\title{
Spherical harmonic analysis of wavefields using multiple circular sensor arrays
}

\author{
Thushara D. Abhayapala, Senior Member, IEEE and Aastha Gupta Student Member, IEEE
}

\begin{abstract}
Spherical harmonic decomposition of wavefields is not only an active problem in acoustic signal processing but also a useful tool in a plethora of applications such as 3D beamforming, direction of arrival estimation, and spatial sound recording. This paper presents a novel array structure consisting of a set of parallel circular arrays of sensors to decompose a wavefield into spherical harmonic components. The new structure presented here provides an alternative design to the traditional spherical microphone arrays with increased flexibility on sensor locations. We use the underlying structure of the wave propagation together with the properties of the associated Legendre functions and the spherical Bessel functions to develop a systematic approach to place circular arrays and construct a hybrid array. As an illustration, we design a fifth order spherical harmonic decomposition array using 57 microphones to operate over a frequency band of an octave and compare it with a spherical array. We use computer simulations to show the performance of the array in a beamforming example.
\end{abstract}

\section{Index Terms}

Spherical array of microphones, spherical harmonics, circular array, beamforming, spatial soundfield, wavefield, wavefield decomposition, acoustic scene analysis

Some parts of the preliminary work related to the current manuscript has been appeared in Proc. of the International Workshop on Acoustic Echo and Noise Control (IWAENC08).

Authors are with the Applied Signal Processing Group, Department of Information Engineering, RSISE, CECS, The Australian National University. e-mail: \{Thushara.Abhayapala, Aastha.Gupta\}@anu.edu.au. 


\section{Spherical harmonic analysis of wavefields using multiple circular sensor arrays}

\section{INTRODUCTION}

Spherical harmonic analysis of three dimensional wavefields is a useful tool in designing signal processing algorithms for beamforming [1]-[4], source localization [5]-[7], acoustic scene analysis [8], [9], spatial soundfield recordings [10], [11], and spatial soundfield reproduction [12]-[14]. The harmonic analysis technique can be used to decompose an observed wavefield into spherical harmonic components by sampling the field using an array of sensors. Whilst spherical microphone arrays [10], [11], [15], [16] have been shown to be a natural choice for spherical harmonic decomposition, there are a number of limitations and constraints which restrict their usefulness. Specifically, the sensor positions of spherical arrays need to meet a strict orthonormality condition resulting in limited flexibility of array geometry. The spherical arrays also suffer from numerical ill conditioning at some frequencies. This paper provides a non-spherical microphone array structure, with increased flexibility, for allowable sensor locations in order to perform spherical harmonic decomposition of wavefields. The proposed array design in this paper can not only be used in acoustic applications but also in other areas such as wireless communications.

The spherical microphone arrays are mainly based on two configurations, the open-sphere where microphones are arranged on free field [10] and the hard-sphere where microphones are arranged around a rigid sphere [11]. The open-sphere configuration with a single sphere suffers numerical ill conditioning at certain frequencies due to zeros of the spherical Bessel functions involved [15]. This problem can be overcome by having concentric spheres [10], [17], [18], a combination of rigid and open spheres [19], [20] and/or measurement of radial velocity [15]. Additionally, the rigid-sphere configuration includes scattering from the sphere which can avoid numerical ill conditioning that is associated with open-sphere configuration. However, for low frequencies, a large hard-sphere is needed to obtain harmonic coefficients, which may not be desirable in practice. In both configurations and their variants, the symmetry of the sphere has been used in designing the spherical harmonic decomposition algorithms. Specifically, the orthonormality property of the spherical harmonics is approximated by placing microphones on the surface of a sphere. Thus, the microphone placement on the sphere needs to satisfy the orthonormality constraint which reduces flexibility of the array geometry. There are number of recent works [16], [18] which provide optimal and flexible placement of microphones on the sphere. However, spherical geometry is still an integral part of these designs. A comprehensive analysis of open and rigid spherical array configurations is given in [15], which also includes the effects of finite order, finite number of microphones, inaccuracies in the positioning of microphones, spatial aliasing, and measurement noise.

There are large number of recent papers on the applications of spherical microphone arrays as included in [21]-[26]. 
In [27], Meyer and Elko proposed a method to extract spherical harmonic coefficients using a circular array of microphones on the $x-y$ plane and a microphone at the origin. This was a novel use of circular arrays as a typical use would be to decompose a soundfield into cylindrical harmonics that are suited for height invariant 2D soundfield analysis. Although, Meyer's work gives some flexibility in controlling the vertical spatial response, fundamentally a 2D array on the $\mathrm{x}-\mathrm{y}$ plane is not sufficient to determine all of the spherical harmonic coefficients for 3D fields. In this work, we extend from [27], to investigate the spherical harmonic decomposition of 3D soundfields and propose a systematic way to build a 3D flexible array structure. The proposed structure consists of a set of circular arrays placed on planes parallel to the x-y plane. The added flexibility and robustness in the structure arise from a careful study of the underlying wave propagation theory, specifically in using properties of the associated Legendre functions and spherical Bessel functions. A study of the underlying physics helps us to analyse the contributions from each spherical harmonic mode from different planes and spatial locations of the array. These new insights guide us to place sensors more appropriately and extract spherical harmonic coefficients whilst avoiding numerical ill-conditioning. The spherical arrays suffer numerical ill conditioning, when sensors are placed in locations where the target spherical harmonic has very little energy. However, in our design, we not only systematically avoid these ill conditions but also provide more flexibility in placing sensors.

In Section II, we outline the theory of spherical harmonic analysis of soundfields. We inspect the underlying structure of the received signal on a circular aperture placed parallel to the x-y plane in Section III. In Section IV, we use the insight gained from the underlying structure to propose a hybrid array structure consisting of parallel circular arrays and sensors on the z-axis. We show the array's capability of operating over a frequency band of an octave in Section V and also provide guidelines on how to extend the bandwidth for several octaves. Finally, we present simulations of a fifth order hybrid array to verify the proposed theory and design.

\section{SOUNDFIELD ANALYSIS}

\section{A. Spherical harmonic expansion}

Consider a point $(r, \theta, \phi)$ within a source free region $\Omega$, where $(r, \theta, \phi)$ are the spherical coordinates with respect to an origin located within $\Omega$. The soundfield at a point $(r, \theta, \phi) \in \Omega$ due to some sources outside of $\Omega$ can be expressed in terms of a spherical harmonic expansion [10] as

$$
S(r, \theta, \phi ; k)=\sum_{n=0}^{\infty} \sum_{m=-n}^{n} \alpha_{n m}(k) j_{n}(k r) Y_{n m}(\theta, \phi)
$$

where $m$ and $n(\geq 0)$ are integers, $\alpha_{n m}(k)$ are the spherical harmonic coefficients of the soundfield, $k=2 \pi f / c$ is the wavenumber, $f$ is the frequency, $c$ is the speed of sound, $j_{n}(\cdot)$ are the spherical Bessel functions of order $n$, and

$$
Y_{n m}(\theta, \phi)=\sqrt{\frac{2 n+1}{4 \pi} \frac{(n-|m|) !}{(n+|m|) !}} P_{n|m|}(\cos \theta) e^{i m \phi}
$$

are the spherical harmonics of order $n$ and degree $m$, which are defined in terms of the associated Legendre functions $P_{n|m|}(\cdot)$ and the exponential functions. Knowing the soundfield over angles on a radius $r$, harmonic coefficients 


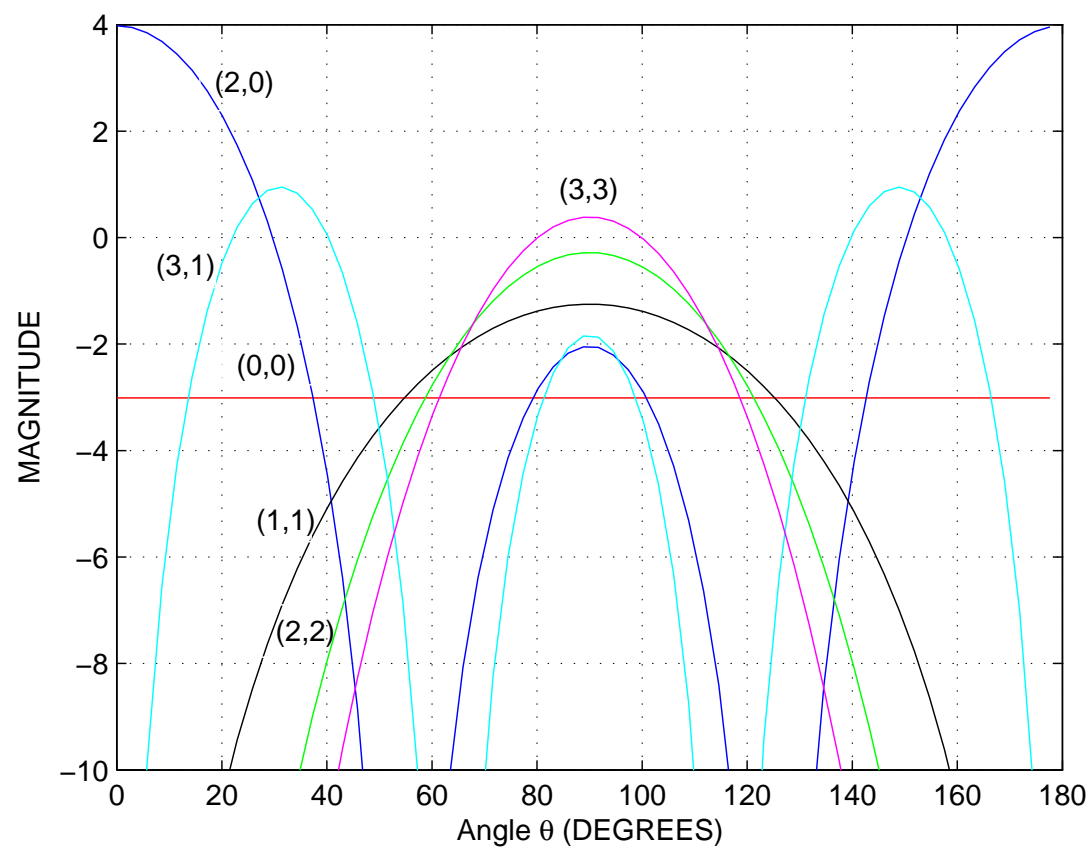

Fig. 1: Magnitude of the normalized associate Legendre functions $\mathcal{P}_{n|m|}(\cos \theta)$ in $\mathrm{dB}$ when $n+|m|$ is even for $(n,|m|)=\{(0,0) ;(2,0) ;(1,1) ;(2,2) ;(3,1) ;(3,3)\}$.

can be calculated using

$$
\alpha_{n m}(k)=\frac{1}{j_{n}(k r)} \int_{0}^{2 \pi} \int_{0}^{\pi} S(r, \theta, \phi ; k) Y_{n m}^{*}(\theta, \phi) \sin \theta d \theta d \phi
$$

provided $j_{n}(k r) \neq 0$. For convenience, we express (1) as

$$
S(r, \theta, \phi ; k)=\sum_{n=0}^{\infty} \sum_{m=-n}^{n} \alpha_{n m}(k) j_{n}(k r) \mathcal{P}_{n|m|}(\cos \theta) E_{m}(\phi)
$$

where $E_{m}(\phi) \triangleq(1 / \sqrt{2 \pi}) e^{i m \phi}$ is the normalized exponential function and

$$
\mathcal{P}_{n|m|}(\cos \theta) \triangleq \sqrt{\frac{2 n+1}{2}} \sqrt{\frac{(n-|m|) !}{(n+|m|) !}} P_{n|m|}(\cos \theta)
$$

is the normalized associated Legendre function. The functions $E_{m}(\phi)$ and $\mathcal{P}_{n|m|}(\cos \theta)$ form orthonormal basis sets in azimuth $\phi \in[0,2 \pi)$ and elevation $\theta \in[0, \pi]$, respectively. The first few normalized associated Legendre functions are illustrated in Figs. 1 and 2. In this paper, we use (4) intuitively to illustrate and design a new array structure to decompose a soundfield into spherical harmonic components.

\section{B. Truncation}

Representation (4) has an infinite number of terms. However, we can truncate this series expansion to a finite number within a given region of interest due to the properties of the Bessel functions (see Fig. 3) and the fact that 


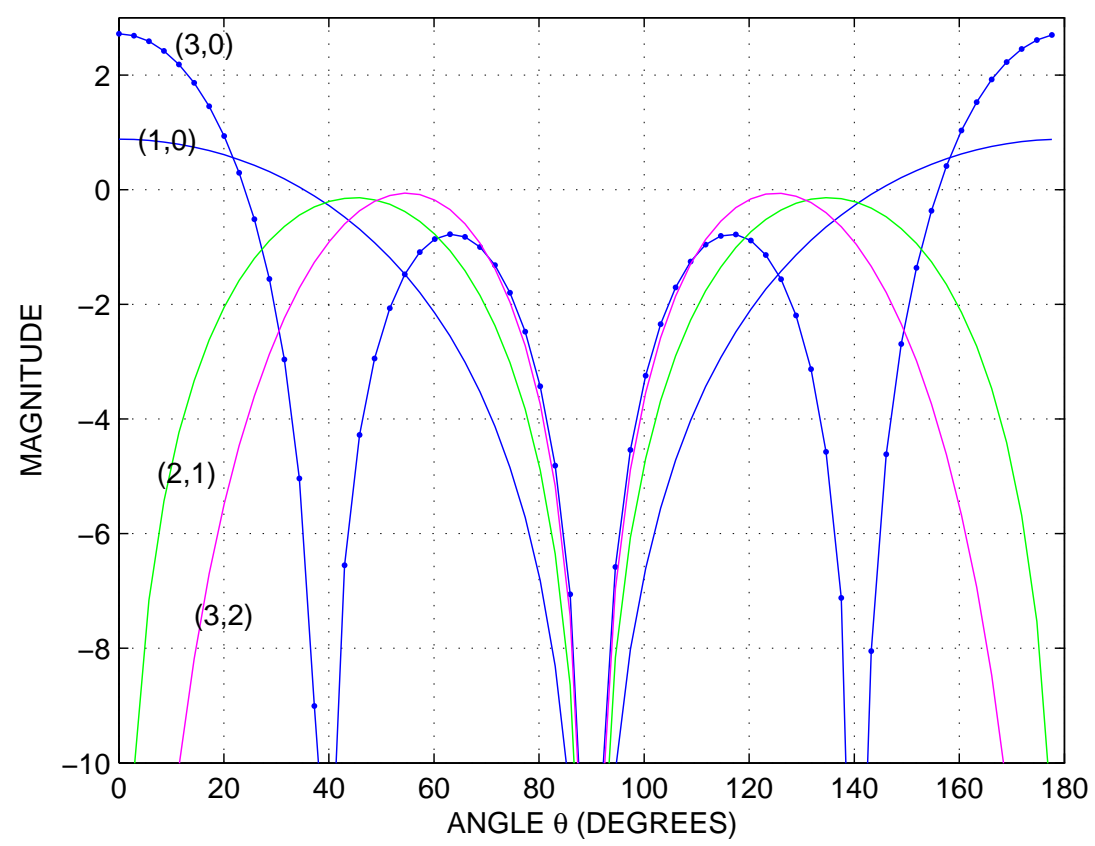

Fig. 2: Magnitude of the normalized associate Legendre functions $\mathcal{P}_{n|m|}(\cos \theta)$ in $\mathrm{dB}$ when $n+|m|$ is odd for $(n,|m|)=\{(1,0) ;(2,1) ;(3,0) ;(3,2)\}$.

the soundfield has to be bounded within a spatial region where all sources are outside [28]. Let $R$ be the radius of the spherical region of interest, then the soundfield inside this sphere can be represented by (4) with summation over $n$ truncated to

$$
N=\left\lceil e^{1} k R / 2\right\rceil
$$

terms [29] with error in truncation equal to less than $67 \%$. Another common rule of thumb for truncation [30, p. 150] of (4) is to use $N=\lceil k R\rceil$. Note that truncation is directly related to the spherical Bessel function $j_{n}(\cdot)$ that has a negligible $(\approx 0)$ magnitude for arguments that are less than the order $n$.

\section{Soundfield Coefficients}

Since we are interested in the soundfield that is restricted to a finite region, then the resulting coefficients of interest are only from the first $(N+1)$ terms. In this case, we can observe from (4) that there are total of $(N+1)^{2}$ coefficients to be determined, which are shown in Table 1 for order $n=0 \ldots N$ and degree $m=-n \ldots n$.

The soundfield coefficients $\alpha_{n m}(k)$ can be estimated by sampling the space using an array of sensors. Spherical microphone arrays approximate the analysis equation (3) by a sum of samples of signal taken over the spherical surface to perform this task [10]. However, there are known limitations of spherical arrays [15] such as the strict orthogonality condition and inflexibility with the sensor geometry. In this paper we develop an alternative array 


\begin{tabular}{c|c|c|c|c|c}
$m \backslash n$ & 0 & 1 & 2 & $\ldots$ & $N$ \\
\hline$N$ & & & & & $\alpha_{N N}$ \\
$\vdots$ & & & & & \\
2 & & & $\alpha_{22}$ & & $\vdots$ \\
1 & & $\alpha_{11}$ & $\alpha_{21}$ & & \\
$m=0$ & $\alpha_{00}$ & $\alpha_{10}$ & $\alpha_{20}$ & $\ldots$ & $\alpha_{N 0}$ \\
-1 & & $\alpha_{1(-1)}$ & $\alpha_{2(-1)}$ & & \\
-2 & & & $\alpha_{2(-2)}$ & & $\vdots$ \\
$\vdots$ & & & & & $\vdots$ \\
$-N$ & & & & & $\alpha_{N(-N)}$
\end{tabular}

TABLE I: Soundfield coefficients arranged with order $n$ and degree $m$. For a $n$th order spherical harmonic system there are $(N+1)^{2}$ coefficients to be determined.

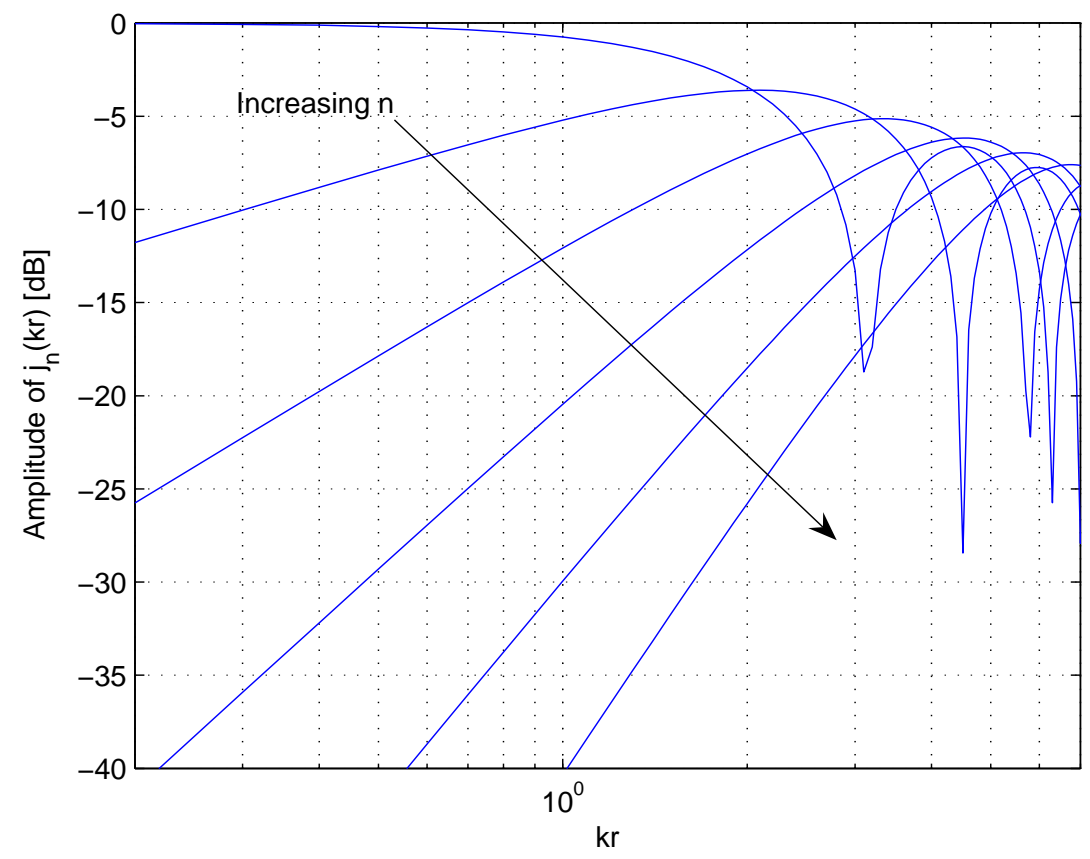

Fig. 3: Magnitude of the spherical Bessel functions $j_{n}(k r)$ of order $n=0,1,2,3,4$, and 5 in $\mathrm{dB}$ showing the characteristics as a function of the argument.

structure to estimate the soundfield coefficients.

\section{Circular Aperture}

In this section, we investigate the soundfield on circles that are parallel to the $\mathrm{x}-\mathrm{y}$ plane. 


\section{A. Azimuth Harmonics}

Let $S\left(r_{q}, \theta_{q}, \phi ; k\right)$ be the the soundfield on a circle given by $\theta=\theta_{q}$ and $r=r_{q}$.

We use (4) to write

$$
S\left(r_{q}, \theta_{q}, \phi ; k\right)=\sum_{n=0}^{N} \sum_{m=-n}^{n} \alpha_{n m}(k) j_{n}\left(k r_{q}\right) \mathcal{P}_{n|m|}\left(\cos \theta_{q}\right) E_{m}(\phi)
$$

where $N=\left\lceil k e^{1} r_{q} / 2\right\rceil$ is from the natural truncation property (see Section II-B). We multiply (7) by $E_{-m}(\phi)$ and integrate with respect to $\phi$ over $[0,2 \pi)$ to get

$$
a_{m}\left(r_{q}, \theta_{q}, k\right)=\sum_{n=|m|}^{N} \alpha_{n m}(k) j_{n}\left(k r_{q}\right) \mathcal{P}_{n|m|}\left(\cos \theta_{q}\right)
$$

where

$$
a_{m}\left(r_{q}, \theta_{q}, k\right) \triangleq \int_{0}^{2 \pi} S\left(r_{q}, \theta_{q}, \phi ; k\right) E_{-m}(\phi) d \phi
$$

Note that the right hand side of (8) is a weighted sum of soundfield coefficients $\alpha_{n m}(k)$ for a given $m$ along a row in Table 1.

The equation (8) can also be evaluated for $m=-N, \ldots, N$, where the truncation number $N$ is dependent on the radius $r_{q}$ of the circle. Also, for a given circle $\left(r_{q}, \theta_{q}\right)$, contribution from some of the spherical harmonic components could be zero if either $j_{n}\left(k r_{q}\right)=0$ or $\mathcal{P}_{n|m|}\left(\cos \theta_{q}\right)=0$. This is clear from Figures 1 and 2, which plot the magnitude of the normalized associated Legendre functions $\mathcal{P}_{n|m|}(\cos \theta)$ in dBs. Hence, we have to be careful while using (8) for coefficient calculations. That leads us to the the main contribution of this paper, which is to show how to extract spherical harmonic coefficients $\alpha_{n m}(k)$ by exploiting (8) from a number of carefully placed circles on different $\left(r_{q}, \theta_{q}\right)$ using the properties of the spherical Bessel functions (Fig. 3) and the associated Legendre functions.

\section{B. Sampling of circles}

Until now we have assumed that the soundfield is known at all points on a given circular aperture. In practice, we can not obtain the soundfield at every point on these circles. In this section, we show how to only use samples of the soundfield on these circles, which enable us to provide design guidelines in order to build practical microphone arrays.

Consider a circular aperture at $\left(r_{q}, \theta_{q}\right)$. To evaluate the integral in (8), we replace the integral with a summation because in practice only a finite number of samples of $S\left(r_{q}, \theta_{q}, \phi ; k\right)$ can be obtained. Recall that due to natural truncation (see Section II-B), at a radius of $r_{q}$ the field is limited to $N_{q}=\left\lceil k e r_{q} / 2\right\rceil$ orders. Thus, the maximum mode $m$ involved is $N_{q}$ and, $S\left(r_{q}, \theta_{q}, \phi ; k\right)$ is mode limited to $N_{q}$, i.e., it contains terms with $e^{j m \phi}$ with $m=-N_{q}, \ldots, N_{q}$. According to Shannon's sampling theorem for periodic functions, $S\left(r_{q}, \theta_{q}, \phi ; k\right)$ can be reconstructed by its samples over $[0,2 \pi]$ with at least $\left(2 N_{q}+1\right)$ samples. Hence, we approximate the integral in (9) by a summation:

$$
a_{m}\left(r_{q}, \theta_{q}, k\right) \approx \frac{2 \pi}{V_{q}} \sum_{v=1}^{V_{q}} S\left(r_{q}, \theta_{q}, \phi_{v} ; k\right) E_{-m}\left(\phi_{v}\right),
$$


where $V_{q} \geq\left(2 N_{q}+1\right)$ are the number of sampling points ${ }^{1}$ on the circle $\left(r_{q}, \theta_{q}\right)$.

\section{Uninspired Least Squares}

Suppose that our goal is to design an $N$ th order microphone array, then we need to estimate $(N+1)^{2}$ spherical harmonic coefficients. To calculate these coefficients, let there be $Q \geq(N+1)$ circles of microphones located on planes given by $\left(r_{q}, \theta_{q}\right), q=1, \ldots, Q$. Now, for a particular value of $m$, we write (8) for all the applicable circles, and obtain a set of simultaneous equations given by

$$
\boldsymbol{J}_{m} \boldsymbol{\alpha}_{m}=\boldsymbol{a}_{m}, \text { for } m=-N, \ldots, N
$$

where

$$
\boldsymbol{J}_{m}=\left[\begin{array}{ccc}
j_{|m|}\left(k r_{1}\right) \mathcal{P}_{|m||m|}\left(\cos \theta_{1}\right) & \cdots & j_{N}\left(k r_{1}\right) \mathcal{P}_{N|m|}\left(\cos \theta_{1}\right) \\
\vdots & \ddots & \vdots \\
j_{|m|}\left(k r_{Q}\right) \mathcal{P}_{|m||m|}\left(\cos \theta_{Q}\right) & \cdots & j_{N}\left(k r_{Q}\right) \mathcal{P}_{N|m|}\left(\cos \theta_{Q}\right)
\end{array}\right],
$$

The harmonic coefficients $\boldsymbol{\alpha}_{m}$ can be calculated by solving the system of linear equations described by (11) for each $m$. Since there will be noise in any measurement, it is necessary to solve (11) in the least squares sense by setting up an over determined system, i.e., number of active circles ${ }^{2}$ being greater than $N-|m|$. If $\left(r_{q}, \theta_{q}\right)$, $q=1, \ldots, Q$ are chosen such that $\boldsymbol{J}_{m}$ has a valid Moore-Penrose inverse $\boldsymbol{J}_{m}^{+}$, then $\boldsymbol{\alpha}_{m}$ can be calculated for each $m$ by solving (11) in the least squares sense as

$$
\boldsymbol{\alpha}_{m}=\boldsymbol{J}_{m}^{+} \boldsymbol{a}_{m}
$$

However, if we choose $\left(r_{q}, \theta_{q}\right)$ arbitrarily there could be a number of singularities in (12). In Section IV, we sample the $3 D$ space by circles to avoid these ill conditions, where we exploit the underlying structure of the wave propagation rather than relying on the ability of the least squares. That is, we propose a set of rules, and guidelines to place circles in 3D space to avoid singularities.

\section{Insight into harmonic structure}

Firstly, by inspecting (8) for specific values of $\left(r_{q}, \phi_{q}\right)$ and $m$,

we find that for a single sensor at the origin, i.e $\left(r_{q}, \phi_{q}\right)=(0,0)$, the only available mode is $m=0$. Hence, we can obtain $\alpha_{00}(k)$ from a single measurement at the origin by

$$
\alpha_{00}(k)=a_{0}(0, \theta, k) / \mathcal{P}_{0|0|}(\cos \theta)=\sqrt{2} a_{0}(0, \theta, k),
$$

\footnotetext{
${ }^{1}$ Note that we can use non-uniform samples on the circles as long as the separation between any two samples are less than the maximum separation required for the Shannon sampling theorem.

${ }^{2}$ There are specific harmonic components present on these circles.
} 


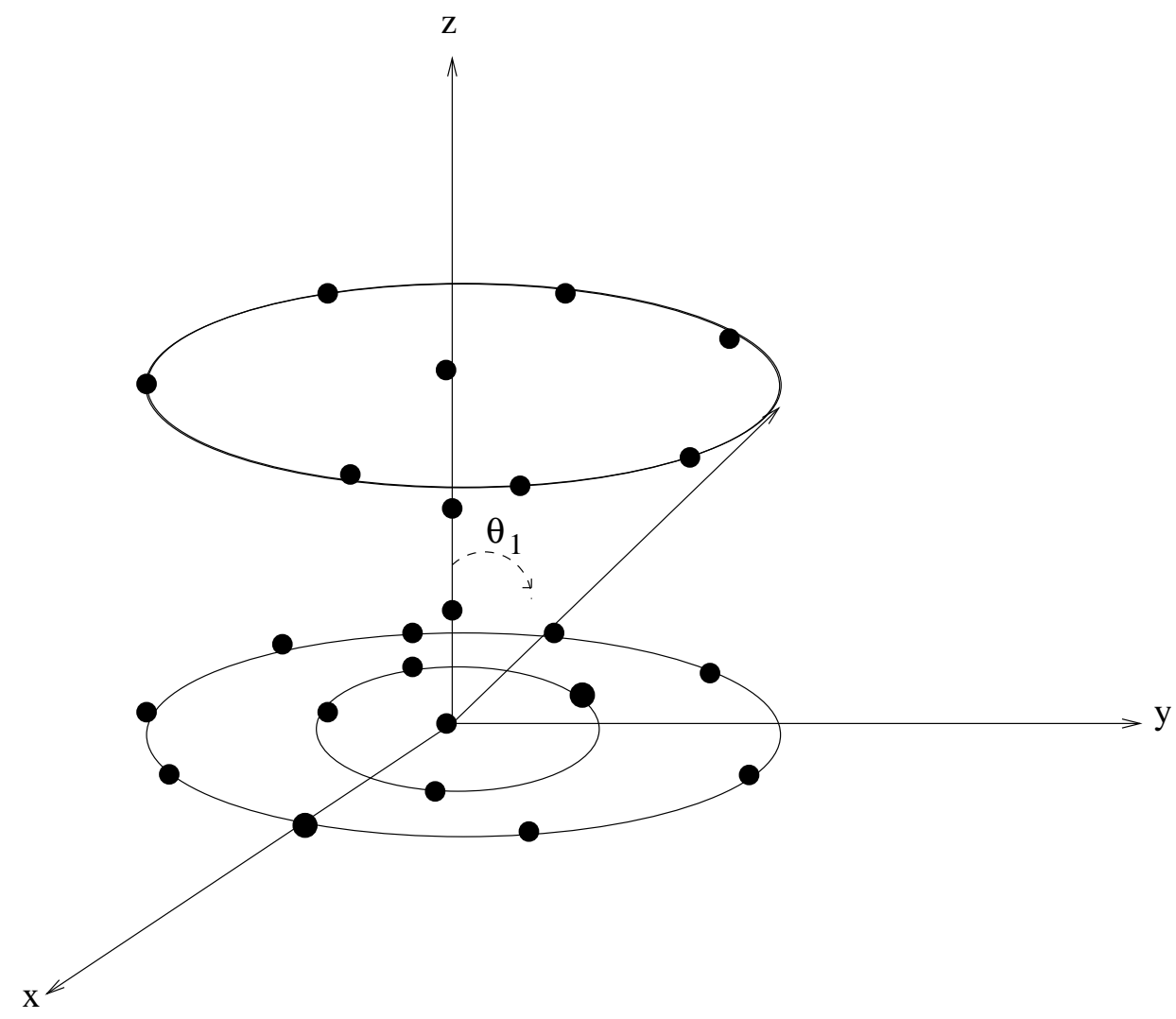

Fig. 4: An example circular array structure for third order spherical harmonic decomposition.

since $\mathcal{P}_{0|0|}(\cos \theta)=1 / \sqrt{2}$.

Secondly, when $\theta=\pi / 2, \mathcal{P}_{n|m|}(\cos (\pi / 2))=0$ if $n+|m|$ is odd. This can be seen from Fig. 2, which depicts the normalized associated Legendre functions up to order 3 for odd $n+|m|$ values. Note that from Fig. 1, for $n+|m|$ even values, the normalized associated Legendre functions have local maxima at $\theta=\pi / 2$. Thus, the corresponding harmonic component do not greatly attenuate at or around $\theta=\pi / 2$.

Thirdly, when $\theta=0$, or $\pi$, the normalized associated Legendre functions have the property,

$$
\mathcal{P}_{n|m|}(\cos \{0, \pi\})=\mathcal{P}_{n|m|}( \pm 1)\left\{\begin{array}{l}
=0, \text { if } m \neq 0, \\
\neq 0, \text { if } m=0 .
\end{array}\right.
$$

Therefore, for points at $\theta=0$ and $\pi$, i.e., on the z-axis, the soundfield only contains the components from $m=0$ spherical harmonics.

These are some of the useful properties that we can observe from (8) using the characteristics of the spherical Bessel functions (Fig. 3), and the normalized associated Legendre functions (Figures 1 and 2). In the next section, we use these properties to construct a 3D array structure for estimating spherical harmonic coefficients of spatial soundfields. 


\begin{tabular}{c|c|c|c}
$N$ & Total number of coefficients & $n+|m|$ even & $n+|m|$ odd \\
\hline 0 & 1 & 1 & 0 \\
1 & 4 & 3 & 1 \\
2 & 9 & 6 & 3 \\
3 & 16 & 10 & 6 \\
4 & 25 & 15 & 10 \\
5 & 36 & 21 & 15 \\
$N$ & $(N+1)^{2}$ & $\frac{(N+1)(N+2)}{2}$ & $\frac{N(N+1)}{2}$
\end{tabular}

TABLE II: List of number of coefficients for $n+|m|$ even and odd for a given $N$ th order spherical harmonic system.

\section{HYBRID ARRAY OF CIRCLES}

In section III-D, we showed that the soundfield coefficients $\alpha_{n m}(k)$, when $n+|m|$ is odd, have zero contribution to the field on the $\mathrm{x}-\mathrm{y}$ plane, whereas all coefficients $\alpha_{n m}(k)$, when $n+|m|$ is even, always have non zero contributions to the soundfield on the x-y plane. For the rest of the paper, we refer $\alpha_{n m}(k)$ with $n+|m|$ even and odd as even coefficients and odd coefficients, respectively. Table II lists the number of even and odd coefficients in a given system with harmonic coeffcients up to the $N$ th order. It is clear that for a given order $N$, there are more even coefficients than odd coefficients. In the following subsection, we give guidelines on how to place a set of circles on the x-y plane to calculate all even coefficients up to the required order $N$.

\section{A. Circles on the $x-y$ plane: Even coefficients}

Our first circle on the $\mathrm{x}-\mathrm{y}$ plane is a circle of zero radius, i.e., a sensor at the origin, to estimate $\alpha_{00}$. For a $N$ th order system, we place another $N / 2$ (when $N$ is even) or $(N+1) / 2$ (when $N$ is odd) circles on the x-y plane. Based on the properties of the Bessel functions, we choose the radii of these circles as

$$
r_{q}=\frac{2}{k_{o}}, \frac{4}{k_{o}}, \ldots, \frac{N}{k_{o}}, \text { for } q=1, \ldots,
$$

where $k_{o}$ is a carefully chosen frequency ${ }^{3}$ within the desired frequency band, i.e., $k_{o} \in\left[k_{\ell}, k_{u}\right]$, where $k_{\ell}$ and $k_{u}$ are the lower and upper frequency band limits, respectively.

With this choice, the soundfield at a frequency $k$ on a circle of radius $r_{q}$ is order limited (see (6)) to

$$
N_{q}(k)=\left\{\begin{array}{l}
\left\lceil N e^{1}\left(k / k_{o}\right)\right\rceil \text { if } q=N / 2 \text { or }(N+1) / 2, \\
\left\lceil 2 q e^{1}\left(k / k_{o}\right)\right\rceil \text { otherwise. }
\end{array}\right.
$$

This property limits the higher order components of the soundfield present at a particular radius $r_{q}$. Also, the lower order components are guaranteed to be present due to the choice of radii in (14) which avoids the Bessel zeros. In Section V, we show how (14) is useful for operation of the array over a broadband of frequencies.

\footnotetext{
${ }^{3}$ We choose $k_{o}$ such that the array can work over a frequency band of an octave. We discuss broadband operation of the array in Section V.
} 
When we evaluate (8) for the circles on the $x-y$ plane, we get a set of a simultaneous system of equations similar to $(11)$ as

$$
\boldsymbol{J}_{m}^{\mathrm{e}} \boldsymbol{\alpha}_{m}^{\mathrm{e}}=\boldsymbol{a}_{m}, \text { for } m=-N, \ldots, N
$$

where

$$
\boldsymbol{J}_{m}^{\mathrm{e}}=\left[\begin{array}{cccc}
j_{|m|}\left(k r_{1}\right) \mathcal{P}_{|m||m|}(0) & j_{|m|+2}\left(k r_{1}\right) \mathcal{P}_{(|m|+2)|m|}(0) & \cdots & j_{N}\left(k r_{1}\right) \mathcal{P}_{N|m|}(0) \\
\vdots & & \ddots & \vdots \\
j_{|m|}\left(k r_{N}\right) \mathcal{P}_{|m||m|}(0) & j_{|m|+2}\left(k r_{N}\right) \mathcal{P}_{(|m|+2)|m|}(0) & \cdots & j_{N}\left(k r_{N}\right) \mathcal{P}_{N|m|}(0)
\end{array}\right],
$$

and

$$
\boldsymbol{a}_{m}=\left[a_{m}\left(r_{1}, \pi / 2, k\right), a_{m}\left(r_{2}, \pi / 2, k\right), \ldots, a_{m}\left(r_{Q}, \pi / 2, k\right)\right]^{T} .
$$

The last column of (17), and the last term of (18) are for a system when both $N$ and $|m|$ are either even or odd integers. Otherwise, $N$ in the corresponding terms should be replaced by $N-1$.

Suppose the highest order $N$ of the system is odd, then the matrix $\boldsymbol{J}_{m}^{\mathrm{e}}$ (17) becomes close to a lower triangular matrix, this is due to the choice of radii $r_{q}$ given by (14). The constraint in (14) forces the higher order harmonic contributions on smaller radii circles to be negligible. Thus, the magnitude of elements in each row of (17) rapidly goes to zero after the diagonal element. Along with the constraint in (14), we also know that $\mathcal{P}_{n|m|}(0) \neq 0$ in (17). Both of these conditions ensure that there is at least one non zero element in each row of (17), thereby making (17) always non singular unlike (12). Also, if the number of circles is equal to the number of unknown even coefficients, then we can solve (16) exactly

$$
\boldsymbol{\alpha}_{m}^{\mathrm{e}}=\left(\boldsymbol{J}_{m}^{\mathrm{e}}\right)^{-1} \boldsymbol{a}_{m}
$$

where $\left(\boldsymbol{J}_{m}^{\mathrm{e}}\right)^{-1}$ is the inverse of $\boldsymbol{J}_{m}^{\mathrm{e}}$.

For the case when $N$ is odd and $m=N$, (11) reduces to a single equation with one unknown $\alpha_{N N}$. However, if we use this equation alone to calculate $\alpha_{N N}$, there may be errors involved as there could be some contribution from $\alpha_{(N+2) N}$.

To improve the accuracy of the calculation, we can include an additional circle after the last circle and include the next higher order even coefficient in the equation (which we can discard once calculated).

Lastly, when $m=0$, we can modify (8) as

$$
a_{0}\left(r_{n}, \pi / 2, k\right)-\alpha_{00}(k) j_{0}\left(k r_{n}\right) \mathcal{P}_{0|0|}(0)=\sum_{n=1}^{N} \alpha_{n 0}(k) j_{n}\left(k r_{q}\right) \mathcal{P}_{n|0|}(0)
$$

since $\alpha_{00}(k)$ is known from the sensor at the origin. Now we can modify (16) accordingly. Alternatively, we can include the sensor at the origin as a circle in (16). Then the first row of (17) would be $[1 / \sqrt{2}, 0,0, \ldots]$. 


\section{B. Sampling on z-axis}

In the previous section, we showed how to calculate even coefficients from circles on the $\mathrm{x}-\mathrm{y}$ plane. In this section, we use another property of the associated Legendre functions, i.e., all the points on the z-axis only contain $m=0$ coefficients, i.e, $\mathcal{P}_{n|m|}( \pm 1)=0$ for $m \neq 0$. Therefore, by placing sensors on the z-axis, we can estimate the coefficients $\alpha_{n 0}$, for $n=1,3,5, \cdots$. Note that $\alpha_{n 0}$, for $n=0,2,4, \cdots$ could be estimated from circles on the $\mathrm{x}-\mathrm{y}$ plane (see Section IV-A).

For a $N$ th order system $(N$ odd), we place sensors on the z-axis at

$$
r_{\mathrm{z} q}=\frac{q}{k_{o}} \text { for } q=1,2, \ldots, N \text {. }
$$

Similar to (14), the above constraint limits the presence of higher order harmonics in the signal received at sensors closer to the origin.

For a sensor at $\left(r_{\mathrm{z} q}, 0\right)$, we can write $(8)$ as

$$
a_{0}\left(r_{\mathrm{zq}}, 0, k\right)=\sum_{n=0}^{N} \alpha_{n 0}(k) j_{n}\left(k r_{\mathrm{zq}}\right) \mathcal{P}_{n|0|}(\cos 0)
$$

Using (5) and [31, p. 688], we obtain ${ }^{4}$

$$
\begin{aligned}
\mathcal{P}_{n|0|}(\cos 0) & =\sqrt{\frac{2 n+1}{2}} P_{n|0|}(1) \\
& =\sqrt{\frac{2 n+1}{2}} .
\end{aligned}
$$

Since $\alpha_{n 0}$ for even orders can be calculated from the procedure in Section IV-A, using (24), we write (23) as

$$
\sum_{n=1, \text { odd } n}^{N} \alpha_{n 0}(k) \sqrt{\frac{2 n+1}{2}} j_{n}\left(k r_{\mathrm{zq}}\right)=a_{0}\left(r_{\mathrm{z} q}, 0, k\right)-\sum_{n=0, \text { even } n}^{N} \alpha_{n 0}(k) \sqrt{\frac{2 n+1}{2}} j_{n}\left(k r_{\mathrm{zq}}\right) .
$$

By evaluating (25) for sensors at $\left(r_{\mathrm{z} q}, 0\right)$ for $q=1,2, \ldots, N$, we have the following system of equations

$$
\boldsymbol{J}_{0}^{\mathrm{z}, \mathrm{o}} \boldsymbol{\alpha}_{0}^{\mathrm{o}}=\boldsymbol{a}_{0}^{\mathrm{z}}-\boldsymbol{J}_{0}^{\mathrm{z}, \mathrm{e}} \boldsymbol{\alpha}_{0}^{\mathrm{e}}
$$

where

$$
\begin{gathered}
\boldsymbol{J}_{0}^{\mathrm{z,o}}=\left[\begin{array}{cccc}
j_{1}\left(k r_{\mathrm{z} 1}\right) \sqrt{3 / 2} & j_{3}\left(k r_{\mathrm{z} 1}\right) \sqrt{7 / 2)} & \cdots & j_{N}\left(k r_{\mathrm{z} 1}\right) \sqrt{(2 N+1) / 2)} \\
\vdots & \ddots & \vdots \\
j_{1}\left(k r_{\mathrm{z} N}\right) \sqrt{3 / 2)} & j_{3}\left(k r_{\mathrm{z} N}\right) \sqrt{7 / 2)} & \cdots & j_{N}\left(k r_{\mathrm{z} N}\right) \sqrt{(2 N+1) / 2)}
\end{array}\right] \\
\boldsymbol{J}_{0}^{\mathrm{z,e}}=\left[\begin{array}{cccc}
j_{0}\left(k r_{\mathrm{z} 1}\right) \sqrt{1 / 2)} & j_{2}\left(k r_{\mathrm{z} 1}\right) \sqrt{5 / 2)} & \cdots & j_{N-1}\left(k r_{\mathrm{z} 1}\right) \sqrt{(2 N-1) / 2)} \\
\vdots & & \ddots & \vdots \\
j_{0}\left(k r_{\mathrm{z} N}\right) \sqrt{1 / 2)} & j_{2}\left(k r_{\mathrm{z} N}\right) \sqrt{5 / 2)} & \cdots & j_{N}\left(k r_{\mathrm{z} N}\right) \sqrt{(2 N-1) / 2)}
\end{array}\right],
\end{gathered}
$$

\footnotetext{
${ }^{4}$ Note that for a sensor at $\left(r_{\mathrm{zq}}, \pi\right), P_{n|0|}(\cos \pi)=P_{n|0|}(-1)=(-1)^{n}$.
} 


\begin{tabular}{c|c|c|c|c}
$N$ & $\begin{array}{c}\text { Total number } \\
\text { of coefficients }\end{array}$ & $\begin{array}{c}\text { Number } \\
\text { from x-y plane }\end{array}$ & $\begin{array}{c}\text { Number } \\
\text { from z-axis }\end{array}$ & $\begin{array}{c}\text { Number from } \\
\text { parallel planes }\end{array}$ \\
\hline 0 & 1 & 1 & 0 & 0 \\
1 & 4 & 3 & 1 & 0 \\
2 & 9 & 6 & 1 & 2 \\
3 & 16 & 10 & 2 & 4 \\
4 & 25 & 15 & 2 & 8 \\
5 & 36 & 21 & 3 & 12 \\
$N$ & $(N+1)^{2}$ & $\frac{(N+1)(N+2)}{2}$ & $\frac{N}{2}$ or $\frac{N+1}{2}$ & $\frac{N^{2}}{2}$ or $\frac{N^{2}-1}{2}$
\end{tabular}

TABLE III: This table lists the number of coefficients that can be calculated from (i) circles on the $x-y$ plane, (ii) sensors on the z-axis, and (iii) from parallel planes to the $x-y$ plane.

$$
\boldsymbol{a}_{0}^{\mathrm{z}}=\left[a_{0}\left(r_{\mathrm{z} 1}, 0, k\right), a_{0}\left(r_{\mathrm{z} 2}, 0, k\right), \ldots, a_{0}\left(r_{\mathrm{zN}}, 0, k\right)\right]^{T},
$$

and

$$
\boldsymbol{\alpha}_{0}^{\mathrm{e}}=\left[\alpha_{00}, \alpha_{20}, \ldots, \alpha_{(N-1) 0}\right]^{T}
$$

Equation (26) can be solved in the least squares sense to obtain

$$
\boldsymbol{\alpha}_{0}^{\mathrm{o}}=\boldsymbol{J}_{0}^{\mathbf{z , o}+} \boldsymbol{a}_{0}^{\mathrm{z}}-\boldsymbol{J}_{0}^{\mathbf{z , o +}} \boldsymbol{J}_{0}^{\mathbf{z , e}} \boldsymbol{\alpha}_{0}^{\mathrm{e}}
$$

where $\boldsymbol{J}_{0}^{\mathrm{z,o}+}$ is the Moore-Penrose inverse of $\boldsymbol{J}_{0}^{\mathrm{z,o}}$.

\section{Circles on parallel planes: Estimating odd coefficients}

So far we have shown how to estimate even spherical harmonics from the $x-y$ plane and odd harmonics with $m=0$ from the sensors on the z-axis. To reiterate, for a $N$ th order system, we have estimated $(N+1)(N+2) / 2$ even coefficients from the x-y plane, and $N / 2$ (even $N)$ or $(N+1) / 2$ coefficients from the z-axis (see Table III). Now, we are left with $N^{2} / 2$ (for even $N$ ) or $\left(N^{2}-1\right) / 2$ (for odd $N$ ) coefficients to be calculated, which we do using circles placed parallel to the $\mathrm{x}-\mathrm{y}$ plane.

It is clear from Table III that we need to have one or more parallel circles for second and higher order systems. We carefully check the patterns of the normalized associate Legendre functions to determine possible values for $\theta_{\mathrm{o} q}$, such that the contribution of odd harmonics is sufficient and non-zero. Figure 5 depicts $\mathcal{P}_{n|m|}(\cos \theta)$ for $n+|m|$ odd when (a) $n-|m|=1$ and (b) $n-|m|=3$. It is evident from these plots that there are clear patterns depending on the difference between $n$ and $|m|$. Based on these figures, one can choose suitable values of $\theta_{\mathrm{o} q}$ to place circles to calculate the remaining odd harmonics. Suggested range of values for $\theta_{\mathrm{o} q}$ are given in the Table IV, which guarantee existence of harmonic contributions of unknown coefficients at these circles.

After choosing $\theta_{\mathrm{o} q}$, we need appropriate values for $r_{\mathrm{o} q}$ to limit the higher order harmonic contribution on a given 


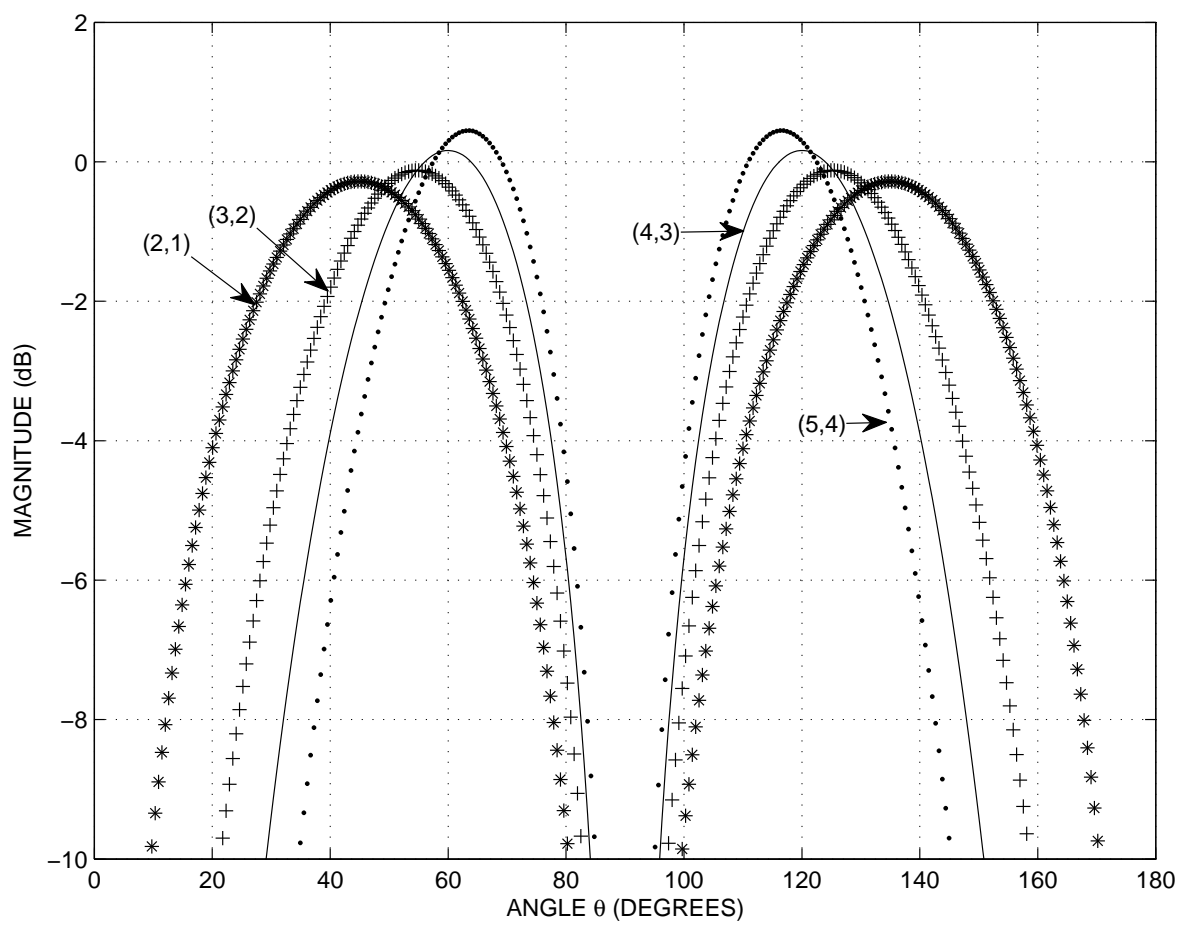

(a)

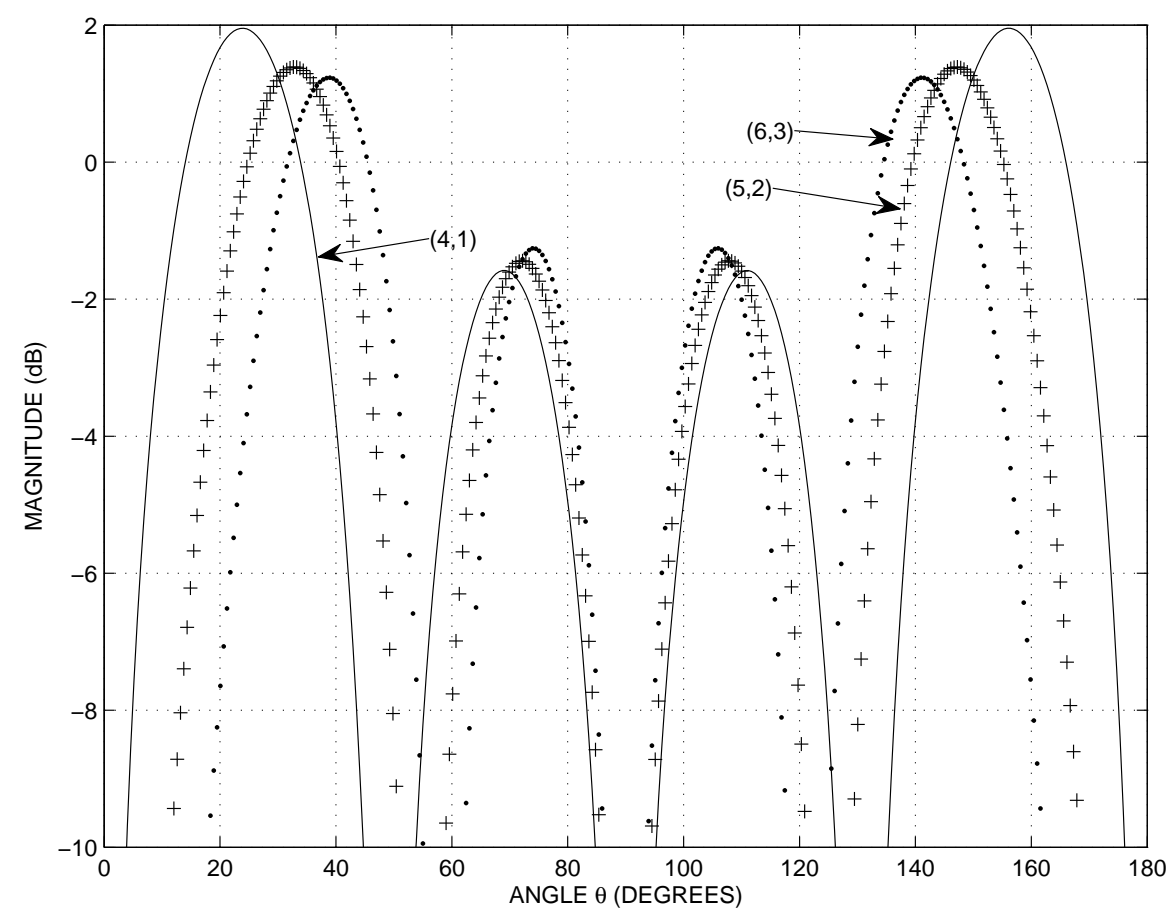

(b)

Fig. 5: Magnitude of the normalized associate Legendre functions $\mathcal{P}_{n|m|}(\cos \theta)$ in $\mathrm{dB}$ (a) when $n-|m|=1$ and (b) when $n-|m|=3$. 


\begin{tabular}{c|c|c}
$n-|m|$ & Range of $\theta_{\mathrm{o} q}$ & $(n,|m|)$ \\
\hline \hline 1 & $50^{\circ}-65^{\circ}$ and $115^{\circ}-130^{\circ}$ & $\begin{array}{c}(2,1),(3,2), \\
(4,3),(5,4), \ldots\end{array}$ \\
\hline 3 & $20^{\circ}-635^{\circ}, 68^{\circ}-72^{\circ}$, & $(4,1),(5,2), \ldots$ \\
& $108^{\circ}-112^{\circ}$ and $145^{\circ}-160^{\circ}$ & \\
\hline 5 & $72^{\circ}-83^{\circ}, 45^{\circ}-50^{\circ}$, & $(6,1),(7,2)$, \\
& $11^{\circ}-21^{\circ}, 97^{\circ}-108^{\circ}$, & $(8,3), \ldots$ \\
& $130^{\circ}-135^{\circ}$, and $159^{\circ}-169^{\circ}$ &
\end{tabular}

TABLE IV: Possible values for $\theta_{\mathrm{oq}}$ to place parallel circles to calculate the odd harmonic coefficients for $m \neq 0$.

circle. Thus, we choose ${ }^{5}$

$$
r_{\mathrm{o} q}=\frac{n}{k_{o}},
$$

where $n$ is the maximum harmonic order targeted to be extracted from the circle and $k_{o}$ is the same as for (14) and (22).

Since we use the methods in Sections IV-A and IV-B to calculate even and $m=0$ coefficients, respectively, we can write (8) as

$$
\sum_{\substack{n=|m| \\ \text { odd } n+|m|}}^{N} \alpha_{n m}(k) j_{n}\left(k r_{\mathrm{o} q}\right) \mathcal{P}_{n|m|}\left(\cos \theta_{\mathrm{o} q}\right)=a_{m}\left(r_{\mathrm{o} q}, \theta_{\mathrm{o} q}, k\right)-\sum_{\substack{n=|m| \\ \text { even } n+|m|}}^{N} \alpha_{n m}(k) j_{n}\left(k r_{\mathrm{oq}}\right) \mathcal{P}_{n|m|}\left(\cos \theta_{\mathrm{o} q}\right)
$$

for $m=-N, \ldots, N$ and $m \neq 0$. By evaluating (34) for $q=1,2, \ldots$ we obtain

$$
\boldsymbol{J}_{m}^{\mathrm{o}} \boldsymbol{\alpha}_{m}^{\mathrm{o}}=\boldsymbol{a}_{m}-\hat{\boldsymbol{J}}_{m}^{\mathrm{e}} \boldsymbol{\alpha}_{m}^{\mathrm{e}}
$$

where

$$
\begin{aligned}
& \boldsymbol{J}_{m}^{\mathrm{o}}=\left[\begin{array}{cccc}
j_{|m|+1}\left(k r_{\mathrm{o} 1}\right) \mathcal{P}_{(|m|+1)|m|}\left(\cos \theta_{\mathrm{o} 1}\right) & j_{|m|+3}\left(k r_{\mathrm{o} 1}\right) \mathcal{P}_{(|m|+3)|m|}\left(\cos \theta_{\mathrm{o} 1}\right) & \cdots & j_{N}\left(k r_{\mathrm{o} 1}\right) \mathcal{P}_{(N-1)|m|}\left(\cos \theta_{\mathrm{o} 1}\right) \\
\vdots & & \ddots & \vdots \\
j_{|m|+1}\left(k r_{\mathrm{o} Q}\right) \mathcal{P}_{(|m|+1)|m|}\left(\cos \theta_{\mathrm{o} Q}\right) & j_{|m|+3}\left(k r_{\mathrm{o} Q}\right) \mathcal{P}_{(|m|+3)|m|}\left(\cos \theta_{\mathrm{o} Q}\right) & \cdots & j_{N}\left(k r_{\mathrm{o} Q}\right) \mathcal{P}_{(N-1)|m|}\left(\cos \theta_{\mathrm{o} Q}\right)
\end{array}\right] \\
& \hat{\boldsymbol{J}}^{\mathrm{e}}{ }_{m}=\left[\begin{array}{cccc}
j_{|m|}\left(k r_{\mathrm{o} 1}\right) \mathcal{P}_{|m||m|}\left(\cos \theta_{\mathrm{o} 1}\right) & j_{|m|+2}\left(k r_{\mathrm{o} 1}\right) \mathcal{P}_{(|m|+2)|m|}\left(\cos \theta_{\mathrm{o} 1}\right) & \cdots & j_{N}\left(k r_{\mathrm{o} 1}\right) \mathcal{P}_{N|m|}\left(\cos \theta_{\mathrm{o} 1}\right) \\
\vdots & & \ddots & \vdots \\
j_{|m|}\left(k r_{\mathrm{o} Q}\right) \mathcal{P}_{|m||m|}\left(\cos \theta_{\mathrm{o} Q}\right) & j_{|m|+2}\left(k r_{\mathrm{o} Q}\right) \mathcal{P}_{(|m|+2)|m|}\left(\cos \theta_{\mathrm{o} Q}\right) & \cdots & j_{N}\left(k r_{\mathrm{o} Q}\right) \mathcal{P}_{N|m|}\left(\cos \theta_{\mathrm{o} Q}\right)
\end{array}\right]^{(3)} \\
& \boldsymbol{\alpha}_{m}^{\mathrm{o}}=\left[\alpha_{(|m|+1) m}, \alpha_{(|m|+3) m}, \ldots, \alpha_{(N-1) m}\right]^{T}, \\
& \boldsymbol{\alpha}_{m}^{\mathrm{e}}=\left[\alpha_{(|m|) m}, \alpha_{(|m|+2) m}, \ldots, \alpha_{N m}\right]^{T} \text {, and } \\
& \boldsymbol{a}_{m}=\left[a_{m}\left(r_{\mathrm{o} 1}, \theta_{\mathrm{o} 1}, k\right), a_{m}\left(r_{\mathrm{o} 2}, \theta_{\mathrm{o} 2}, k\right), \ldots, a_{m}\left(r_{\mathrm{o} Q}, \theta_{\mathrm{o} Q}, k\right)\right]^{T} .
\end{aligned}
$$

\footnotetext{
${ }^{5}$ This is a guide only. The exact design values of radii can be around the suggested quantities.
} 
It can be seen that for a second order system $(N=2)$, only 2 coefficients $\left(\alpha_{21}\right.$, and $\left.\alpha_{2(-1)}\right)$ need to be determined from this method. An appropriately placed circle is sufficient to calculate these two coefficients. For both of these coefficients $n-|m|=1$. Hence, from Table IV, $\theta_{01}$ can be selected. Since the maximum $n$ is $2, r_{01}=2 / k_{o}$ is the appropriate radial distance to the circle.

Whereas, for a fifth order system $(N=5)$, there are 12 odd coefficients to be estimated from parallel circles. There are two sets of $n-|m|$ values that are involved: (i) $n-|m|=1$ and (ii) $n-|m|=3$. Thus, we can choose $\theta_{\mathrm{o} 1}$ and $\theta_{\mathrm{o} 2}$ according to Table IV. Since the maxima for $n$ involved are 4 and 5 , we can choose $r_{\mathrm{o} 1}=4 / k_{o}$ and $r_{\mathrm{o} 2}=5 / k_{o}$, respectively.

In this section, we have provided guidelines on how to estimate spherical harmonic coefficients by multiple circular sensor arrays placed on and parallel to the $\mathrm{x}-\mathrm{y}$ plane, and sensors on the z-axis. However, we have commented little on the array performance over a desired band of frequencies. In the next section, we will show that by suitably setting $k_{o}$, we can make the array operate for a range of frequencies over an octave.

\section{BROADBAND PERFORMANCE}

The spherical harmonic decomposition method proposed in this paper is reliant on constructing matrices $\boldsymbol{J}^{\mathrm{e}}$, $\boldsymbol{J}^{z, 0}$, and $\boldsymbol{J}^{\mathrm{o}}$ by appropriately placing circular arrays on and parallel to the x-y plane. We call these matrices Bessel matrices. The performance of the system is dependent on the non-singular nature of the Bessel matrices. Until now, we chose $\theta_{q}$ and $r_{q}$ such that Bessel matrices are closer to lower triangular matrices and thus are non singular. However, these matrices are also dependent on the operating frequency.

In the following subsection, we choose the design parameter $k_{o}$ such that the design can work over a band of an octave. In subsection V-B, we show how to extend the array to work over several octaves by using the nested array concept.

\section{A. Design over an octave}

Let $\left[k_{\ell}, 2 k_{\ell}\right]$ be the desired frequency band of an octave. Recall that in the previous sections, we chose different radii $r_{q}$ to place circles which were in terms of a parameter $k_{o}$. The challenge is to choose $k_{o} \in\left[k_{\ell}, 2 k_{\ell}\right]$ such that we can preserve the desirable properties of the Bessel matrices over the frequency band $\left[k_{\ell}, 2 k_{\ell}\right]$. That is for any $k \in\left[k_{\ell}, 2 k_{\ell}\right]$, each of the Bessel matrix is non singular and closer to a lower triangular matrix. In other words, we need to consider a range of values, for which the spherical Bessel function of a given order has sufficient amplitude greater than the noise threshold, but the the subsequent spherical Bessel functions do not.

In this paper, we choose $k_{o}=k_{\ell} e^{1} / 2$, which approximately satisfies our requirements. This is illustrated by calculating values of $j_{n}(z)$ for $z=2 n / e^{1}$ and $z=4 n / e^{1}$, as tabulated in Table V. In the Bessel matrices, the argument of the spherical Bessel function is $k r_{q}$. We have placed circles such that $r_{q}=n / k_{o}$, where $n$ is the order of interest. Then, we have $k r_{q}=n\left(k / k_{0}\right)$. By choosing $k_{o}=k_{\ell} e^{1} / 2$ gives us $k r_{q}=2 n k /\left(k_{\ell} e^{1}\right)$. Hence, for the lower and upper end of the octave, this argument is given by $2 n / e^{1}$ and $4 n / e^{1}$, respectively. These are the values 


\begin{tabular}{c|c|c|c||c|c|c}
$n$ & $2 n / e^{1}$ & $j_{n}\left(2 n / e^{1}\right)$ & $j_{n+1}\left(2 n / e^{1}\right)$ & $4 n / e^{1}$ & $j_{n}\left(4 n / e^{1}\right)$ & $j_{n+1}\left(4 n / e^{1}\right)$ \\
\hline 1 & 0.7358 & 0.2322 & 0.0347 & 1.4715 & 0.3922 & 0.1233 \\
2 & 1.4715 & 0.1233 & 0.0269 & 2.9430 & 0.2957 & 0.1466 \\
3 & 2.2073 & 0.0776 & 0.0200 & 4.4146 & 0.2413 & 0.1542 \\
4 & 2.943 & 0.0529 & 0.0151 & 5.8861 & 0.1994 & 0.1539 \\
5 & 3.6788 & 0.0378 & 0.0115 & 7.3576 & 0.1628 & 0.1481
\end{tabular}

TABLE V: Values of the spherical Bessel functions $j_{n}(z)$ and $j_{n+1}(z)$ for $z=2 n / e^{1}$ and $z=4 n / e^{1}$ which correspond to the lower and upper end of the design frequency band.

as tabulated in Table V for $n=1,2, \ldots, 5$. In Section VI, we simulate our design to show its performance over an octave.

Note that the above explanation is true for any chosen octave regardless of the value of $k_{\ell}$. However, the physical array has different dimensions for different octave bands since we place circles according to $r_{q}=n / k_{o}=2 n /\left(k_{\ell} e^{1}\right)$.

\section{B. Nested array}

To extend the design for few octaves, we can always design a new array structure for each octave and cascade them together. Due to our choice of radii $r_{q}$, we can reuse some of the circular arrays with additional sensors for upper octaves. As an example, for a 5 th order system, we place circles on the $\mathrm{x}$-y plane at $2 / k_{o}, 4 / k_{o}$, and $5 / k_{o}$, according to (14). When we extend the array for the next octave, we can reuse the circle at $2 / k_{o}$ as the second circle of the new array. So the idea is to reuse existing circles for lower frequency bands in higher octaves resulting in a nested array. The concept of nested arrays are not new and have been used in literature for broadband linear arrays [32].

\section{DESIGN EXAMPLE}

We now consider an example of the spherical harmonic extraction array outlined in this paper. Specifically, we design a 5 th order system capable of operating over a frequency band of an octave $\left[k_{\ell}, 2 k_{\ell}\right]$.

We first place three circles on the x-y plane at $r_{1}=2 / k_{o}, r_{2}=4 / k_{o}$, and $r_{3}=5 / k_{o}$ according to (14) with 7 , 11 , and 13 sensors $^{6}$, where $k_{o}=k_{\ell} \exp (1) / 2$. Using (22), five sensors are placed on the z-axis at $1 / k_{o}, 2 / k_{o}, 3 / k_{o}$, $4 / k_{o}$, and $5 / k_{o}$ from the origin. Finally, we position three additional circular arrays at $\left(2 / k_{o}, \pi / 3\right),\left(4 / k_{o}, \pi / 3\right)$, and $\left(5 / k_{o}, \pi / 6\right)$ in accordance with (33) and Table IV. The number of sensors on these parallel circles are 3,11 and 7 , respectively. Thus, we use a total of 57 sensors for the fifth order spherical harmonic extraction array. This is not a unique design as we could have chosen a different set of parameters within the same guidelines.

Our chosen frequency band (an octave) for the simulation is $3000 \mathrm{~Hz}$ to $6000 \mathrm{~Hz}$, however the array design and the results are independent of the frequency band and hold for any octave. For the chosen octave, $k_{\ell}=2 \pi f_{\ell} / c=55.44$,

\footnotetext{
${ }^{6}$ We use two additional sensors than minimum required according to sampling theorem.
} 


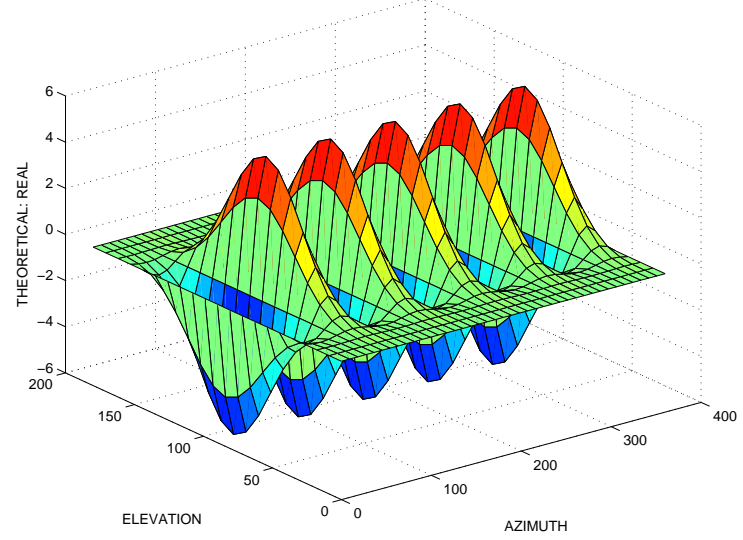

(a)

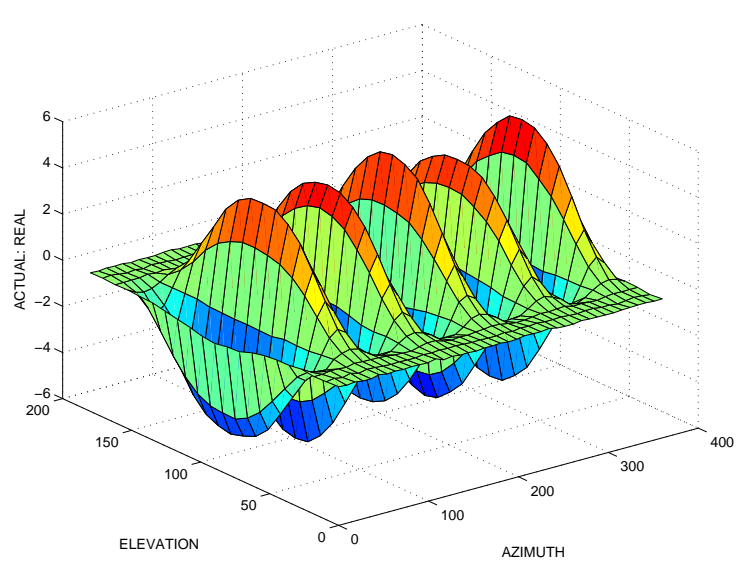

(c)

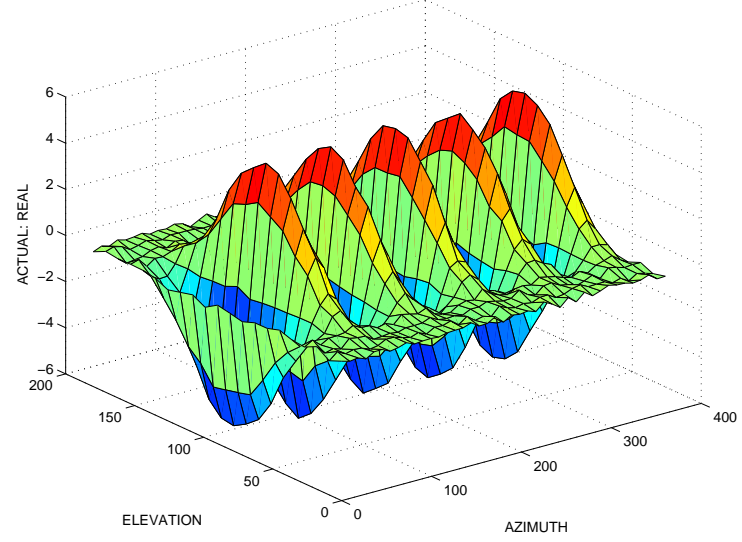

(b)

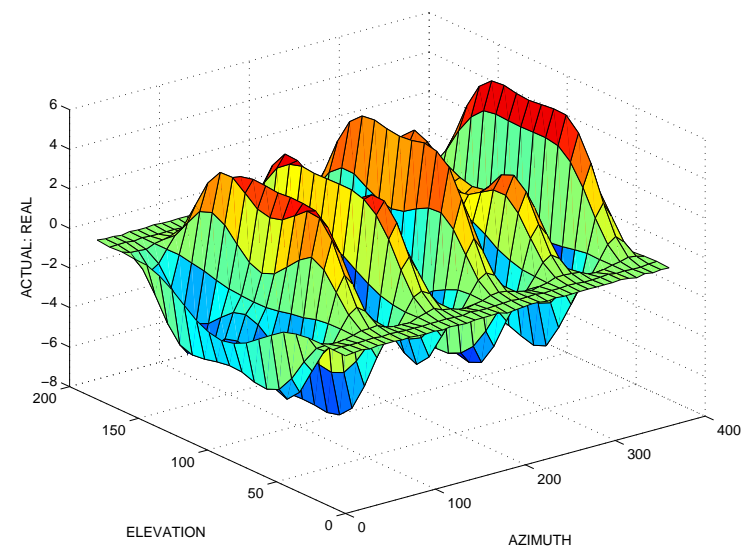

(d)

Fig. 6: Estimated harmonic coefficient $\alpha_{55}$ for a plane wave sweeping over entire 3D space: (a) Theoretical pattern (b) at $f=3000 \mathrm{~Hz}, \mathrm{SNR}=40 \mathrm{~dB}$, (c) at $f=4500 \mathrm{~Hz}, \mathrm{SNR}=40 \mathrm{~dB}$, and (d) at $f=6000 \mathrm{~Hz}, \mathrm{SNR}=40 \mathrm{~dB}$.

where we assume the speed of sound $c=340 \mathrm{~m} / \mathrm{s}$. Unless stated otherwise, we apply a $40 \mathrm{~dB}$ signal to noise ratio (SNR) at each sensor, where the noise is additive white Gaussian (AWGN).

To verify the design objectives, the array was used to estimate all 36 spherical harmonic coefficients $\alpha_{n m}(k)$ from soundfields created by a plane wave sweeping over the entire 3D space. This was performed for all frequencies within the desired octave. The real and imaginary parts of $\alpha_{n m}(k)$ were plotted against the azimuth and elevation of the sweeping plane wave for the lower, mid, and upper ends of the frequency band. As an overview, from the 36 coefficients, we only show $\alpha_{55}(k)$ and $\alpha_{53}(k)$ in Figs. 6 and 7, respectively. It is also seen that at the lower and upper ends of the band, the harmonic patterns get distorted compared to the theoretical value. All the coefficients estimated from the circles on the $\mathrm{x}-\mathrm{y}$ plane (i.e., even coefficients) also maintain their shape for the whole band. There are a few higher order odd coefficients which tend to get distorted towards the edge of the band. The distortion at the lower end of the band is due to the signal power of the harmonic component being lower as compared to 


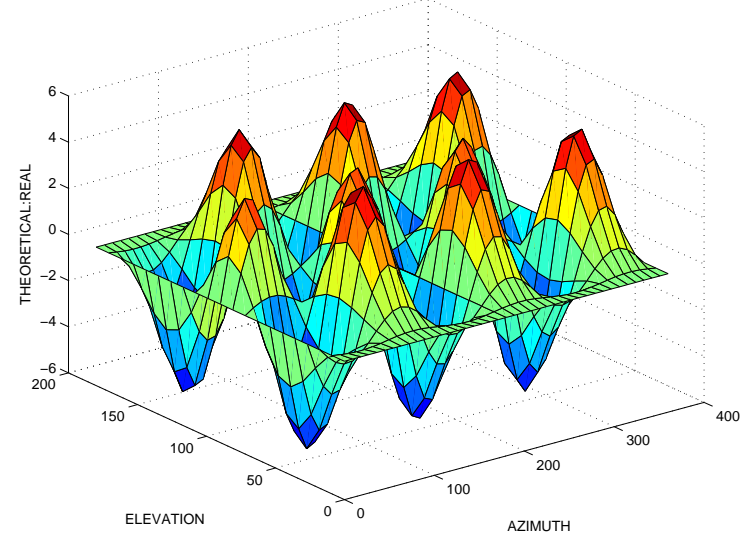

(a)

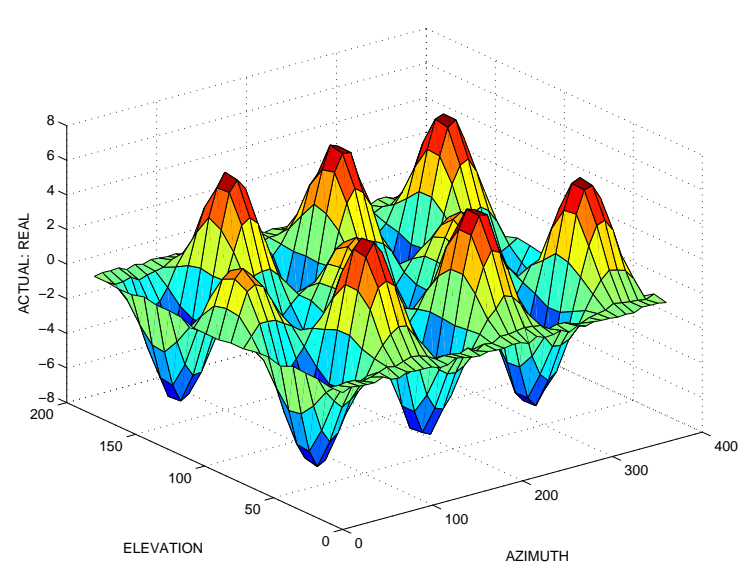

(c)

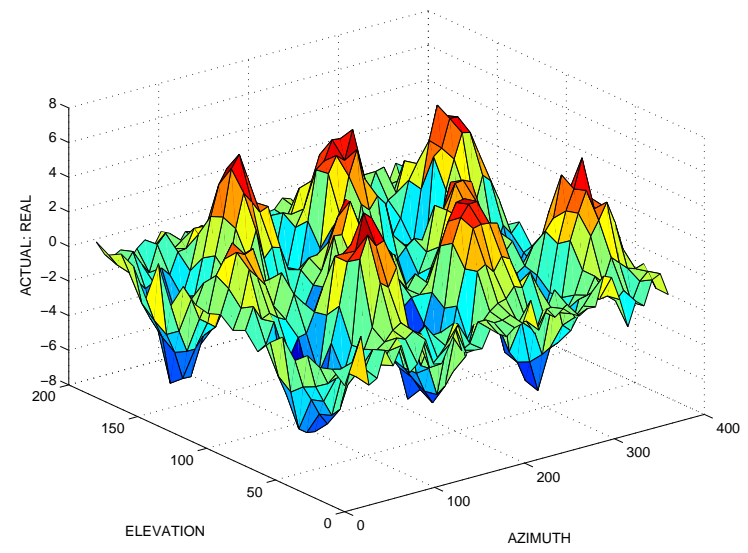

(b)

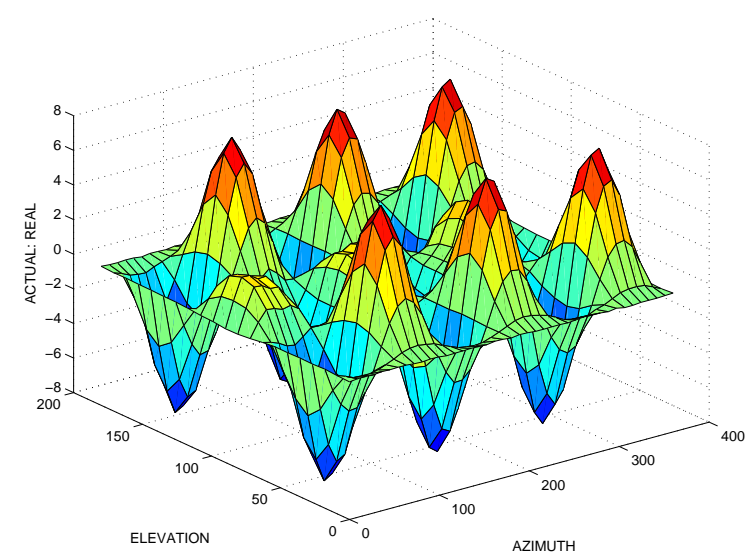

(d)

Fig. 7: Estimated harmonic coefficient $\alpha_{53}$ for a plane wave sweeping over entire 3D space: (a) Theoretical pattern (b) at $f=3000 \mathrm{~Hz}, \mathrm{SNR}=40 \mathrm{~dB}$, (c) at $f=4500 \mathrm{~Hz}, \mathrm{SNR}=40 \mathrm{~dB}$, and (d) at $f=6000 \mathrm{~Hz}, \mathrm{SNR}=40 \mathrm{~dB}$

the noise power, whereas the distortion at the upper end of the band is due to contributions from the higher order harmonic coefficients (greater than 5).

To accurately quantify the performance of the array, we define a Mean Squared Error (MSE) measure as

$$
\operatorname{MSE}_{n m}(k)=\sqrt{\frac{\sum_{p}\left|\alpha_{n m}^{\mathrm{e}}(k)_{p}-\alpha_{n m}(k)_{p}\right|^{2}}{\sum_{p}\left|\alpha_{n m}(k)_{p}\right|^{2}}}
$$

where $\alpha_{n m}^{\mathrm{e}}(k)_{p}$ and $\alpha_{n m}(k)_{p}$ are the extracted and the theoretical spherical harmonic coefficients from the $p$ soundfield (in the example above, a plane wave soundfield from the $p$ th direction), respectively. The MSE measure of the designed hybrid circular array was calculated for all coefficients over plane wave soundfields originating from 800 different directions in 3D space and at frequency intervals of $25 \mathrm{~Hz}$ spanning the bandwidth of [3000,6000] Hz. The results are depicted in Fig. 8a, where the $(n, m)$ th spherical harmonic coefficient corresponds to the coefficient 
number $n^{2}+n+m+1$ on the $\mathrm{x}$-axis ${ }^{7}$.

For comparison, we designed a fifth order open spherical sensor array with 49 sensors using the guidelines given in [10], [17]. The number of sensors needs to be a square number in order to preserve the orthogonality condition. We use [33] to find the locations of sensors on the sphere and the corresponding weightings to approximate the integration in the orthonormality condition. It is impossible to find a suitable radius for the spherical array to extract all harmonics over an octave. We used a sphere with radius of $4 \mathrm{~cm}$, which corresponds to $k_{\ell} r=$ $2 \pi \times 3000 \times 0.04 / 340=2.22$. Note from Fig. 3 , the corresponding $k r$ range $[2.22,4.44]$ for the desired octave has a Bessel zero of the zeroth order.

As for the hybrid circular array, the MSE measure (40) for the 49 sensor spherical array is shown in Fig. 8b. In terms of MSE, both designs have comparable performance for all coefficients except $(0,0)$ coefficient over the desired frequency band. The spherical array could not be used to estimate $(0,0)$ coefficient at some frequencies due to a Bessel zero.

The advantage of the hybrid circular array is in the flexibility in choosing sensor locations. The fifth order hybrid circular array design given in this section is not the only solution. There are other sets of radii and elevation angles that one can choose from. Additionally, the azimuth angle separation between sensors on the circular arrays does not have to be uniformly spaced. Also, a small perturbation to geometric parameters of the hybrid circular array does not have a significant difference to the performance, whereas small perturbations to a spherical array destroys the orthogonality condition.

Finally, to illustrate an application of the fifth order hybrid circular array, we use the harmonic components to construct a beamformer. Once a soundfield/wavefield is decomposed into spherical harmonics, it is straight forward to form beams in 3D. There are a number of references available in the literature [1], [3], [34], [35], which use spherical harmonic decomposition (also termed as modal decomposition) for beamformer design. A farfield beamformer pointing to the direction $\left(\hat{\theta}_{0}, \hat{\phi}_{0}\right)$ can be realized by a weighted addition of the spherical harmonic components of the wavefield [11], [35] as

$$
y(k)=\sum_{n=0}^{\infty} \sum_{m=-n}^{n} \alpha_{n m}(k)\left(i^{-n} /(2 n+1)\right) \mathcal{P}_{n|m|}\left(\cos \hat{\theta}_{0}\right) E_{m}\left(\hat{\phi}_{0}\right) .
$$

We use (41) to form a farfield beamformer in the look direction of $\left(\theta_{0}, \phi_{0}\right) \equiv\left(90^{\circ}, 90^{\circ}\right)$. The response of the hybrid circular array is depicted in Fig. VI at (a) $f=3000 \mathrm{~Hz}$, (b) $f=4500 \mathrm{~Hz}$, and (c) $f=6000 \mathrm{~Hz}$, where the SNR at each sensor is $40 \mathrm{~dB}$. The beamformer maintains its look direction over the entire octave, however there is some degradation of the pattern at the two ends with respect to sidelobe levels. To show the performance of the beamformer over different SNRs, in Figure 10 we plot the response of the beamformer as a function of azimuth angle $\phi$ at different SNR levels for a plane wave impinging from a fixed elevation angle $\theta=90^{\circ}$ at $6000 \mathrm{~Hz}$.

${ }^{7}$ i.e., MSE of $\alpha_{5(-3)}$ is given by the point $n^{2}+n+m+1=5^{2}+5-3+1=28$ on the x-axis. 


\section{CONCLUSION}

In this paper, we have developed a hybrid array structure to decompose a wavefield into spherical harmonic components. The structure consists of (i) a set of concentric circular arrays on the $\mathrm{x}-\mathrm{y}$ plane to calculate spherical harmonic coefficients $\alpha_{n m}$ when $n+|m|$ is even, (ii) a set of individual sensors on z-axis to calculate coefficients $\alpha_{n 0}$ when $m=0$, and (iii) another set of circular arrays placed parallel to the $\mathrm{x}-\mathrm{y}$ plane to calculate remaining $\alpha_{n m}$ when $n+|m|$ is odd. The structure provides an alternative to the traditional spherical microphone arrays to decompose a wavefield into the spherical harmonics. The design can work for a given frequency band of an octave and can be extended to operate over several octaves using the nested array concept. At a lower end of the design band, the performance of the array may degrade due to noise effects, while at higher frequencies presence of higher order harmonics may cause performance reduction. An extra addition of a circular array than the suggested design can improve the performance at both ends of the design frequency band. We have demonstrated the application of the method in a farfield broadband beamformer example over the design octave, whose response is robust to a range of sensor noise levels.

\section{REFERENCES}

[1] R.A. Kennedy, T. Abhayapala, D.B. Ward, and R.C. Williamson, "Nearfield broadband frequency invariant beamforming," in IEEE International Conference on Acoustics, Speech, and Signal Processing, 1996, vol. 2, pp. 905-908.

[2] D.B. Ward and G.W. Elko, "Mixed nearfield/farfield beamforming: a new technique for speechacquisition in a reverberant environment," in IEEE Workshop on Applications of Signal Processing to Audio and Acoustics, New Paltz, NY, USA, 1997.

[3] T.D. Abhayapala, R.A. Kennedy, and R. C. Williamson, "Nearfield broadband array design using a radially invariant modal expansion," The Journal of the Acoustical Society of America, vol. 107, no. 1, pp. 392-403, 2000.

[4] Z. Li and R. Duraiswami, "A robust and self-reconfigurable design of spherical microphone array for multi-resolution beamforming," in IEEE International Conference on Acoustics, Speech and Signal Processing, 2005, vol. 4, pp. 1137-1140.

[5] T.D. Abhayapala and H. Bhatta, "Coherent broadband source localization by modal space processing," in 10th International Conference on Telecommunications, 2003, vol. 2, pp. 1617-1623.

[6] H. Teutsch and W. Kellerman, "Estimation of the number of wideband sources in an acoustic wave field using eigen-beam processing for circular apertures," in IEEE Workshop on Applications of Signal Processing to Audio and Acoustics, 2005, pp. 110-113.

[7] S. Argentieri, P. Danes, and P. Soueres, "Modal analysis based beamforming for nearfield or farfield speaker localization in robotics," in IEEE International Conference on Intelligent Robots and Systems, 2006, pp. 866-871.

[8] Z. Li, R. Duraiswami, E. Grassi, and L.S. Davis, "A spherical microphone array system for traffic scene analysis," in 7th International IEEE Conference on Intelligent Transportation Systems, 2004, pp. 338-342.

[9] A. O'Donovan, R. Duraiswami, and D. Zotkin, "Imaging concert hall acoustics using visual and audio cameras," in IEEE International Conference on Acoustics, Speech and Signal Processing, April 2008, pp. 5284-5287.

[10] T.D. Abhayapala and D.B. Ward, "Theory and design of higher order sound field microphones using spherical microphone array," in IEEE International Conference on Acoustics, Speech and Signal Processing, May 2002, vol. 2, pp. 1949-1952.

[11] J. Meyer and G. Elko, "A highly scalable spherical microphone array based on an orthonormal decomposition of the soundfield," in IEEE International Conference on Acoustics, Speech and Signal Processing, May 2002, vol. 2, pp. 1781-1784.

[12] D.B. Ward and T.D. Abhayapala, "Reproduction of a plane-wave sound field using an array of loudspeakers," IEEE Trans. on Speech Audio Processing, vol. 9, no. 6, pp. 697-707, September 2001.

[13] M.A. Poletti, "Three-dimensional surround sound systems based on spherical harmonics," Journal of Audio Engineering Society, vol. 53, no. 11, pp. 1004-1025, 2005.

[14] T. Betlehem and T.D. Abhayapala, "Theory and design of sound field reproduction in reverberant rooms," The Journal of the Acoustical Society of America, vol. 117, no. 4, pp. 2100-2111, April 2005. 
[15] B. Rafaely, "Analysis and design of spherical microphone arrays," IEEE Transactions on Speech and Audio Processing, vol. 13, no. 1, pp. 135-143, January 2005.

[16] Z. Li, R. Duraiswami, and L.S. Davis, "Flexible layout and optimal cancellation of the orthonormality error for spherical microphone arrays," in IEEE International Conference on Acoustics, Speech and Signal Processing, May 2004, vol. 4, pp. 41-44.

[17] I. Balmages and B. Rafaely, "Open sphere designs for spherical microphone arrays," IEEE Trans. Audio, Speech, and Language Proc., vol. 15, no. 2, pp. 727-732, Ferbruary 2007.

[18] B. Rafaely, "The spherical shell microphone array," IEEE Trans. on Acoustics Speech and Signal Processing., vol. 16, no. 4, pp. 740-747, 2008.

[19] C. Jin, A. Parthy, and A. A. Schaik, "Optimisation of co-centred rigid and open spherical microphone arrays," in 120th Convention of the Audio Engineering Society, Paris, France, May 2006, pp. 388-391.

[20] T.D. Abhayapala and M.C.T. Chan, "Limitation and error analysis of spherical microphone arrays," in 14th International Congress on Sound and Vibration, Cairns, Australia, July 2007.

[21] B.N. Gover, J.G. Ryan, and M.R. Stinson, "Measurements of directional properties of reverberant sound fields in rooms using a spherical microphone array," The Journal of the Acoustical Society of America, vol. 116, no. 4, pp. 2138-2148, 2004.

[22] B. Rafaely, "Plane-wave decomposition of the sound field on a sphere by spherical convolution," The Journal of the Acoustical Society of America, vol. 116, pp. 2149, 2004.

[23] Z. Li, R. Duraiswami, and N.A. Gumerov, "Capture and recreation of higher order 3d sound fields via reciprocity," in Conference of International Community for Auditory Display, 2004, pp. 6-9.

[24] M. Park and B. Rafaely, "Sound-field analysis by plane-wave decomposition using spherical microphone array," The Journal of the Acoustical Society of America, vol. 118, pp. 3094, 2005.

[25] H. Teutsch and W. Kellermann, "Detection and localization of multiple wideband acoustic sources based on wavefield decomposition using spherical apertures," in IEEE International Conference on Acoustics, Speech and Signal Processing, April 2008, pp. 5276-5279.

[26] D.N. Zotkin, R. Duraiswami, and N.A Gumerov, "Sound field decomposition using spherical microphone arrays," in IEEE International Conference on Acoustics, Speech and Signal Processing, 2008, pp. 277-280.

[27] J. Meyer and G. Elko, "Spherical harmonic modal beamforming for an augmented circular microphone array," in IEEE International Conference Acoustics, Speech and Signal Processing,, March 2008, pp. 5280-5283.

[28] R. A. Kennedy, P. Sadeghi, T. D. Abhayapala, and H.M. Jones, "Intrinsic limits of dimensionality and richness in random multipath fields," IEEE Trans. Signal Processing, vol. 55, pp. 2542-2556, June 2007.

[29] T.D. Abhayapala, T.S. Pollock, and R.A. Kennedy, "Characterization of 3d spatial wireless channels," in IEEE 58th Vehicular Technology Conference, Melbourne, February 2003, vol. 1, pp. 123-127.

[30] N.A. Gumerov and R. Duraiswami, Fast multipole methods for the Helmholtz equation in three dimensions, Elsevier, Oxford, UK, 2004.

[31] E. Skudrzyk, The Foundations of Acoustics: Basic Mathematics and Basic Acoustics, Springer-Verlag, New York, 1971.

[32] W. Kellermann, "A self-steering digital microphone array," International Conference on Acoustics, Speech, and Signal Processing, pp. 3581-3584, 1991.

[33] J. Fliege, "The distribution of points on the sphere and corresponding cubature formulae," IMA Journal of Numerical Analysis, vol. 19, no. 2, pp. 317-334, 1999 .

[34] M. Guillaume and Y. Grenier, "Sound field analysis based on analytical beamforming," EURASIP Journal on Applied Signal Processing, , no. 1, pp. 189-189, 2007.

[35] B. Rafaely and M. Kleider, "Spherical microphone array beam steering using wigner-d weighting," IEEE Signal Processing Letters, vol. 15, pp. 417-420, 2008. 


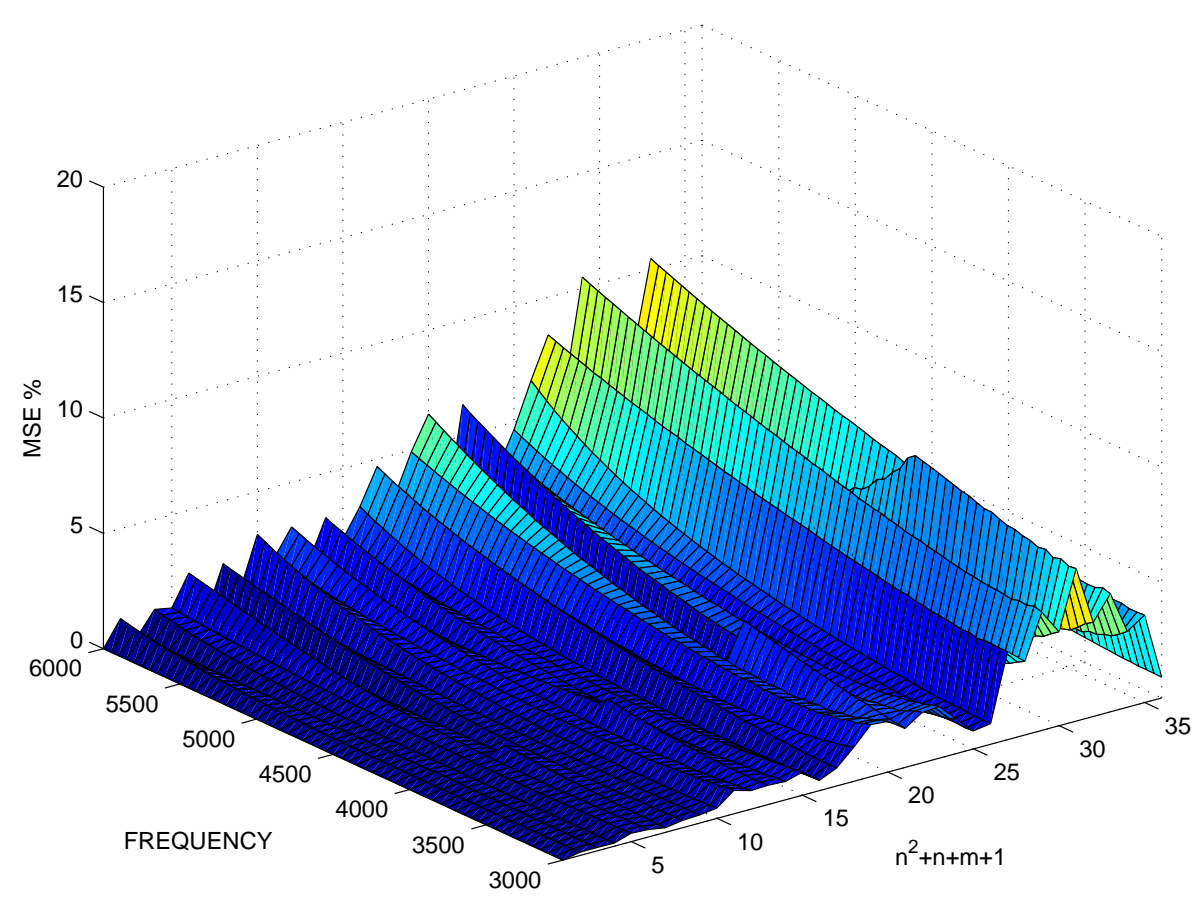

(a)

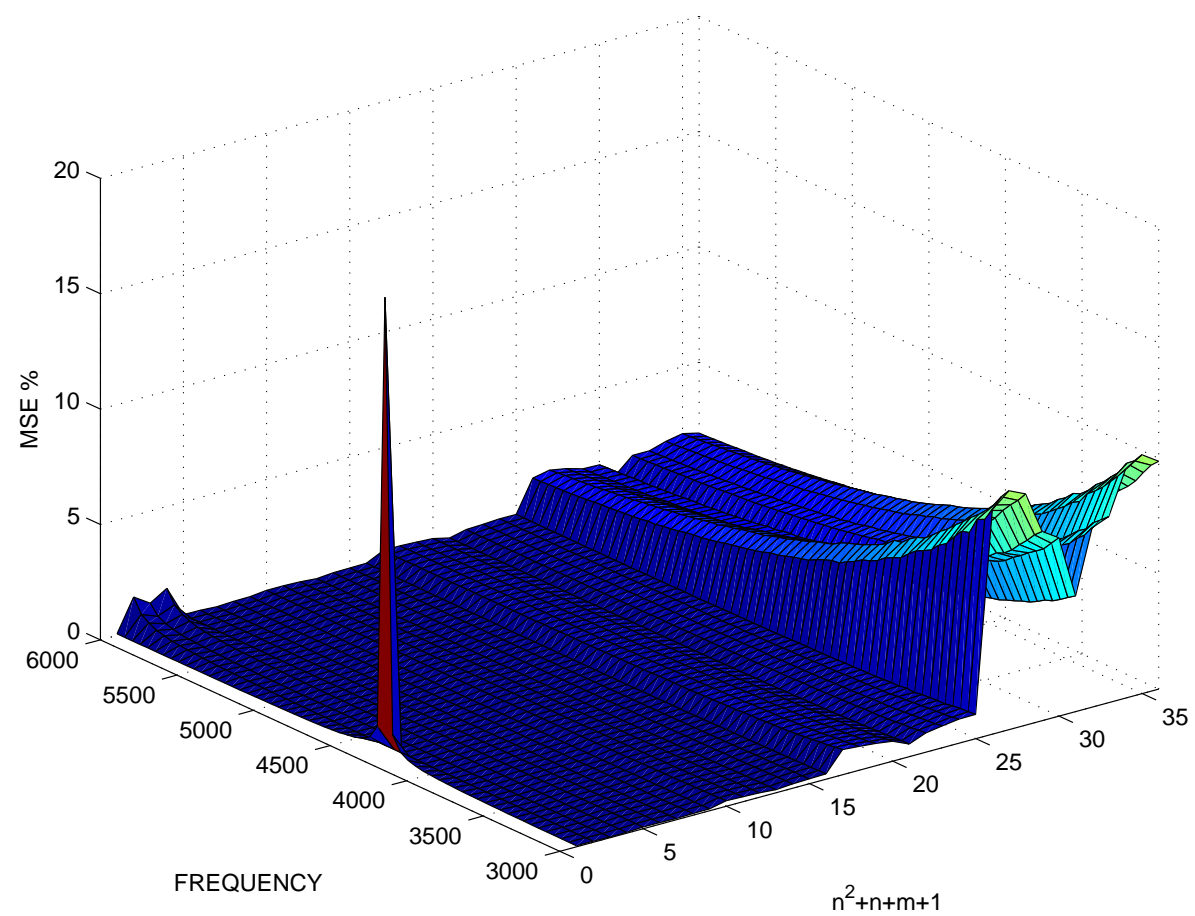

(b)

Fig. 8: The Mean Square Error (MSE) performance measure (40) of the 5th order (a) hybrid circular array (b) spherical array. The soundfields were from plane waves originating from 800 different directions in 3D space and at frequency intervals of $25 \mathrm{~Hz}$ spanning the bandwidth of [3000,6000] Hz. The coefficient number $n^{2}+n+\underset{\text { DRAFT }}{\text { November 16, 2009 }}$
. on the x-axis represents $(n, m)$ harmonic coefficient. 


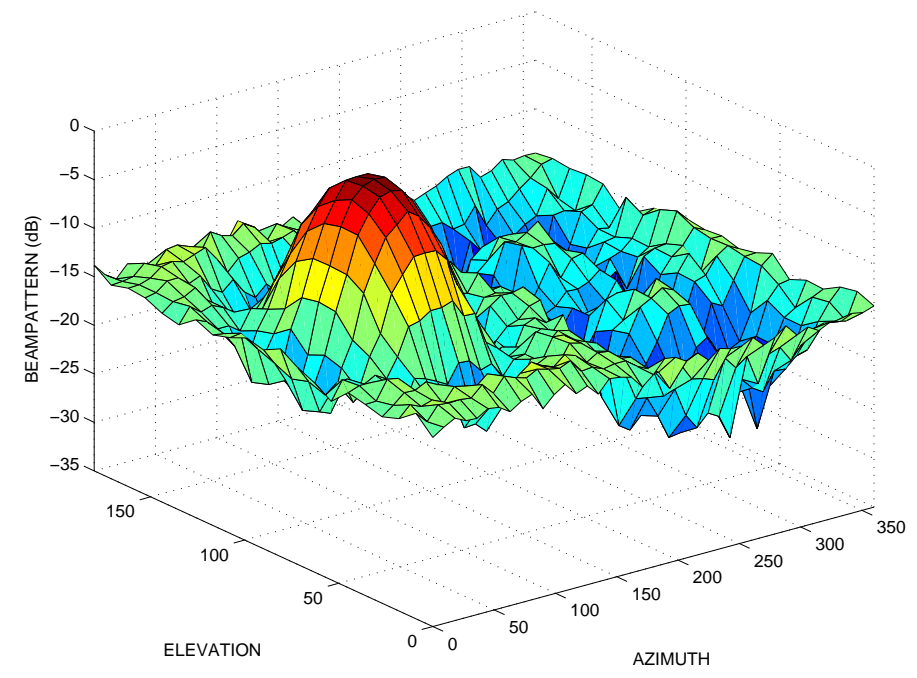

(a)

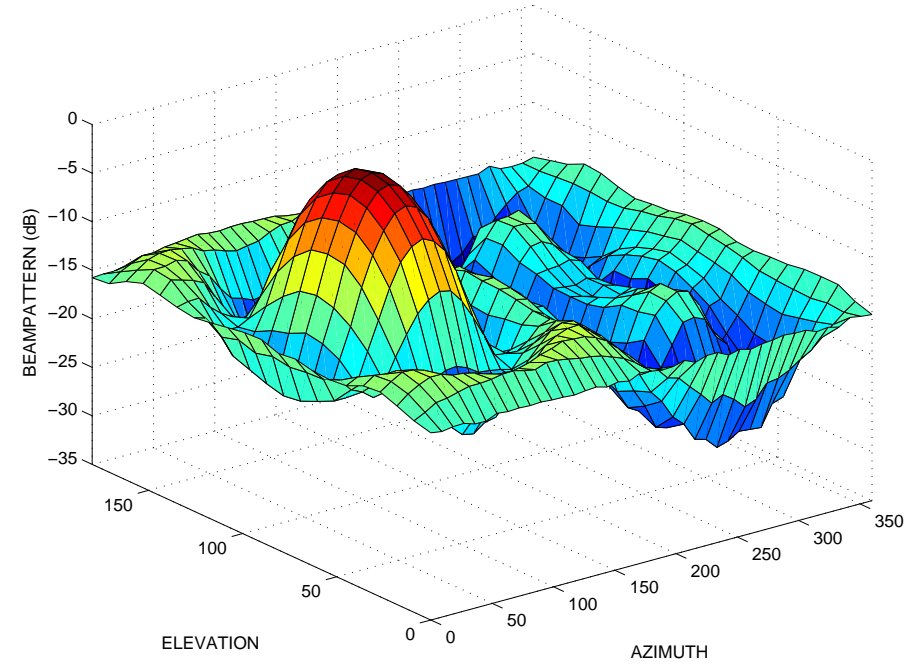

(b)

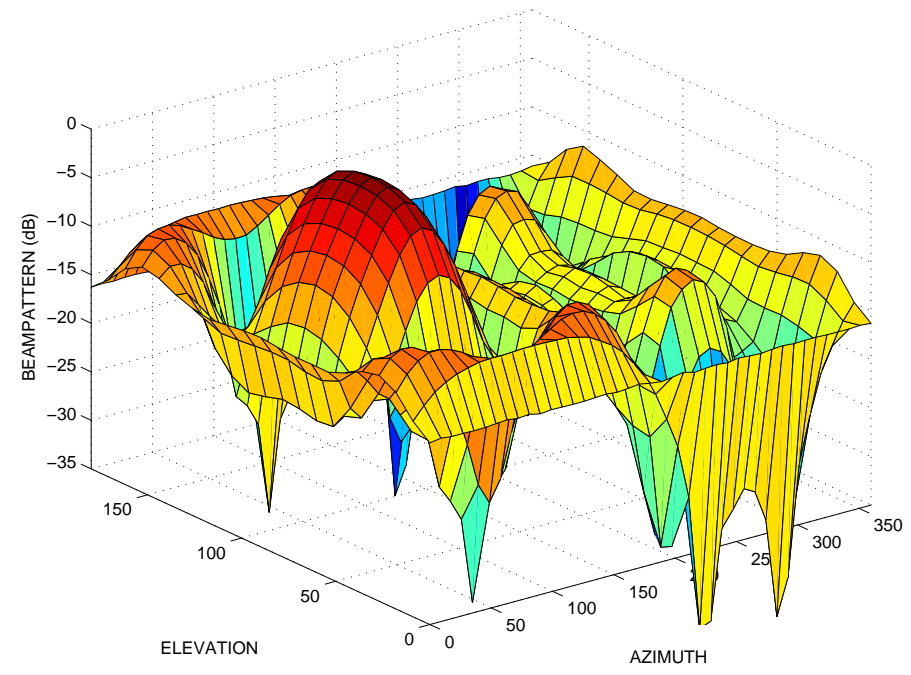

(c)

Fig. 9: Response of the fifth order beamformer design in Section VI at (a) $f=3000$, (b) $f=4500$, and (c)

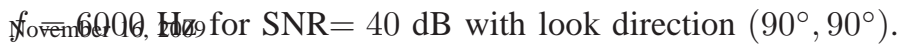




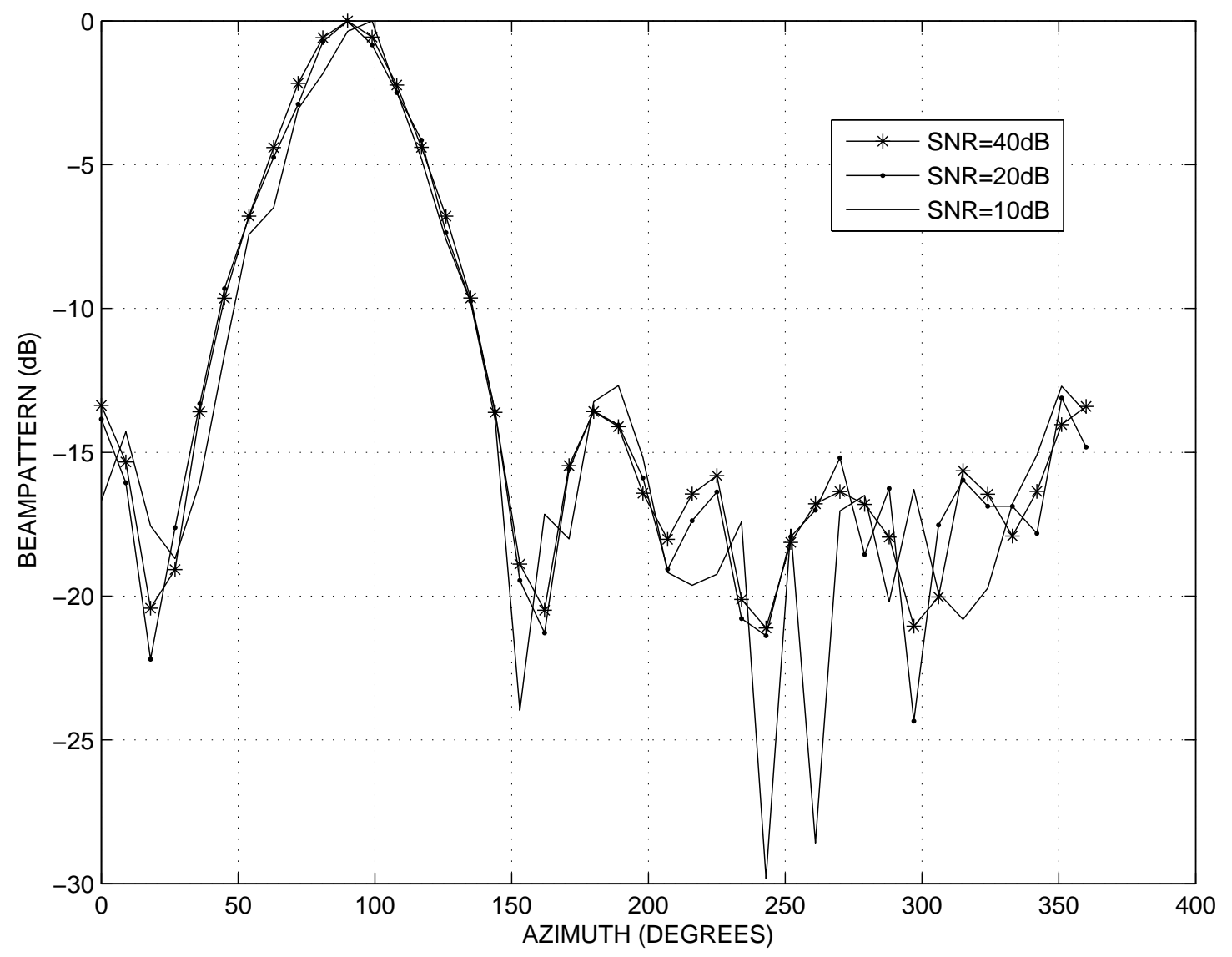

Fig. 10: Response of the fifth order beamformer at $\mathrm{SNR}=40 \mathrm{~dB}, 20 \mathrm{~dB}$, and $10 \mathrm{~dB}, f=6000 \mathrm{~Hz}$, and the look direction $\left(90^{\circ}, 90^{\circ}\right)$. 